ARTICLE

\title{
An oncogenic role of Agrin in regulating focal adhesion integrity in hepatocellular carcinoma
}

Sayan Chakraborty¹, Manikandan Lakshmanan, Hannah L.F. Swa1, Jianxiang Chen¹,2, Xiaoqian Zhang ${ }^{1}$, Yan Shan Ong ${ }^{1}$, Li Shen Loo', Semih Can Akıncılar', Jayantha Gunaratne', Vinay Tergaonkar, Kam M. Hui ${ }^{1,2} \&$ Wanjin Hong ${ }^{1}$

Hepatocellular carcinoma (HCC) is one of the leading causes of cancer-related deaths globally. The identity and role of cell surface molecules driving complex biological events leading to HCC progression are poorly understood, hence representing major lacunae in HCC therapies. Here, combining SILAC quantitative proteomics and biochemical approaches, we uncover a critical oncogenic role of Agrin, which is overexpressed and secreted in HCC. Agrin enhances cellular proliferation, migration and oncogenic signalling. Mechanistically, Agrin's extracellular matrix sensor activity provides oncogenic cues to regulate Arp2/3-dependent ruffling, invadopodia formation and epithelial-mesenchymal transition through sustained focal adhesion integrity that drives liver tumorigenesis. Furthermore, Agrin signalling through Lrp4-muscle-specific tyrosine kinase (MuSK) forms a critical oncogenic axis. Importantly, antibodies targeting Agrin reduced oncogenic signalling and tumour growth in vivo. Together, we demonstrate that Agrin is frequently upregulated and important for oncogenic property of $\mathrm{HCC}$, and is an attractive target for antibody therapy.

\footnotetext{
${ }^{1}$ Institute of Molecular and Cell Biology, Agency for Science, Technology and Research (A`STAR), 61, Biopolis Drive, Proteos, Singapore 138673, Singapore. ${ }^{2}$ Laboratory of Cancer Genomics, Cellular and Molecular Research Division, National Cancer Center Singapore, 11, Hospital drive, Singapore 169610,

Singapore. Correspondence and requests for materials should be addressed to W.H. (email: mcbhwj@imcb.a-star.edu.sg).
} 
epatocellular carcinoma (HCC) is one of the most common malignancies having a high mortality rate worldwide ${ }^{1}$. The cell surface proteins defining various signalling pathways in HCC are not very well characterized ${ }^{2}$, and current therapies are largely restricted to targeting receptor tyrosine kinases ${ }^{3}$. Hence, identification of cell surface proteins of HCC is necessary to broaden the potential of targeted therapy. Although cell surface biotinylation followed by proteomic analysis of enriched proteins offers a useful strategy to screen differentially expressed targets in many cancers, a thorough investigation is lacking for $\mathrm{HCC}^{4,5}$. Here we utilized surface biotinylation to enrich plasma membrane proteins in a stable isotope labelled amino acids in culture (SILAC) ${ }^{6}$-based quantitative proteomic strategy in $\mathrm{Hep} 3 \mathrm{~B}$ hepatoma cell line and non-tumorigenic liver MIHA cells to identify differentially expressed proteins.

Among many proteins showing enriched expression in Hep3B versus MIHA cells, we have focused and characterized Agrin in detail as histopathological analyses indicated its accumulation in liver cirrhosis and induced HCC, cholangiocarcinomas and malignant HCC lesions without a defined role ${ }^{7-9}$. Splice variants of Agrin, a $\sim 210-\mathrm{kDa}$ proteoglycan, are expressed either as membrane protein or secreted in extracellular matrix $(\mathrm{ECM})^{10,11}$. Neural Agrin clusters acetylcholine receptors at synaptic clefts and maintains functional neuromuscular junctions ${ }^{12-14}$. Agrin is expressed in several tissues including muscles and neurons ${ }^{10}$. Agrin binds to Lipoprotein-related receptor 4 (Lrp4), a member of low density lipoprotein receptor (LDLR) family and mediates muscle-specific receptor tyrosine kinase (MuSK) signalling at neuromuscular junctions ${ }^{15,16}$. Neural and muscle Agrins also bind laminin in the ECM, which is involved in cytoskeletal rearrangements and neuronal outgrowths with mechanisms remaining unclear ${ }^{17-19}$.

Integrins and focal adhesion (FA) kinases (FAKs)-associated microenvironment, membrane protrusions and degradation of ECM are not only essential for cancer cell invasion, epithelialmesenchymal transition (EMT) and metastasis but also for promoting tumour growth ${ }^{20,21-27}$. However, not many ECM proteins except collagen can modulate invasiveness and metastasis ${ }^{23}$, and the role of Agrin in regulating oncogenesis is unknown.

Herein we report that Agrin promotes liver carcinogenesis as an ECM sensor regulating cell proliferation, motility, invasiveness, FA integrity and EMT through Lrp4-MuSK signalling axis. In vivo, short hairpin RNA (shRNA)-mediated Agrin depletion or function blocking antibodies suppressed oncogenic signalling and liver tumorigenesis. Collectively, our findings establish a role of Agrin upregulation in HCC and suggest it as an attractive therapeutic target.

\section{Results}

SILAC proteomics identifies differentially expressed proteins. To identify potential diagnostic and/or therapeutic targets differentially expressed in HCC, biotinylated cell surface proteins enriched for plasma membrane fractions were affinity purified with streptavidin sepharose beads. Compared with non-tumorigenic liver MIHA cells, we observed a significant number of proteins enriched in Hep3B and/or HepG2 (HCC) cell lines (Supplementary Fig. 1a). The enrichment of plasma membrane fractions were confirmed by examining the distribution of a membrane marker (integrin $\beta 1$ ) and cytosolic marker (Rho-GDI; Supplementary Fig. 1b). For quantitative evaluation, we combined surface biotinylation and extraction of cell surface proteins and identified them by SILAC-based quantitative mass spectrometry (Fig. 1a). To increase the confidence of protein quantification, only proteins with at least two ratio counts were considered for follow-up analysis. A protein density plot was generated using the ratios of those quantified proteins to determine the thresholds for clustering differentially expressed proteins. Using 10\%, 90\% and in-between quantile-based thresholds, the proteins were categorized as down-, up- and unregulated proteins, respectively (Fig. 1b). In this study, we focussed on proteins that are overexpressed in Hep3B cells and are potential candidates overexpressed in liver carcinoma (red cluster, Fig. 1b). As such, the upregulated protein cluster (Supplementary Table 1) was subjected to Gene Ontology Cellular Component analysis that revealed that $>50 \%$ of upregulated proteins are enriched as plasma membrane proteins (Fig. 1c and Supplementary Table 2). The overexpressed proteins including epidermal growth factor receptor, glypicans and epithelial cell adhesion molecule (EpCAM) have widespread roles in numerous cancers, thereby supporting the authenticity of our SILAC screen. Proteoglycan Agrin was also identified in this screen as an overexpressed surface protein in Hep3B cells (Supplementary Tables 1 and 2).

Validation of selected set of identified proteins. To independently confirm our mass spectrometry findings, biotinylated surface proteins in MIHA and Hep3B cells were affinity purified and the expression levels of selected candidate(s) were analysed by immunoblot analysis. Consistent with our SILAC observations, Agrin, EpCAM, epidermal growth factor receptor and glypican-3 showed increased cell surface expression in Нер3B compared with MIHA cells (Fig. 1d). Moreover, higher expression of these proteins was also evident in the whole-cell lysates of HepG2 and Hep3B cell lines (Fig. 1d). Conversely, sorting nexin5 (SNX5), a protein indicated to be downregulated in Hep3B cell surface has reduced expression in both surface-enriched fraction and total cell lysate (Fig. 1d). The sodium/potassium ATPase $\left(\mathrm{Na}^{+} / \mathrm{K}^{+}\right.$ATPase), a plasma membrane protein and many other transporter proteins with unaffected SILAC ratios did not show significant change between MIHA and Hep3B cells in western blot analysis (Fig. 1d). $\beta$-Actin levels indicate similar loading of whole-cell lysates (Fig. 1d).

Agrin is overexpressed and secreted in HCC cell lines. Among the identified molecules, we considered Agrin as an attractive target in HCC owing to its accumulation in cirrhotic liver and HCC but with little known roles ${ }^{7-9}$. Compared with MIHA cells, Agrin was well expressed in a panel of HCC cell lines with relatively higher levels in metastatic MHCC-97H, MHCC-LM3, Sk-HEP-1 and SNU-449 cells (Fig. 1e). Since secreted neural Agrin aggregates acetylcholine receptors, we next examined whether it is secreted in cancer cell lines and hence can potentially act as a biomarker. Supernatants of HCC cell lines (MHCC-LM3 and Hep3B), a breast cancer cell line SkBr-3 and control MIHA cells were tested for Agrin secretion. Indeed, secreted Agrin was high in HCC cell culture supernatants, low in SkBr-3 and hardly detectable in MIHA cells (Fig. 1f).

In vivo, mouse xenografts also showed a higher expression of Agrin in the liver (Hep3B) tumours compared with MCF7 cell breast carcinoma (Fig. 1g). These results show an elevated expression and secretion of Agrin in HCC cell lines and Hep3B xenografts.

Lipid raft-enriched Agrin is constitutively internalized. Reported lipid raft localization of neural Agrin prompted us to examine the exact membrane localization of Agrin in HCC cell lines $^{28}$. Indeed, the bulk of cell surface-bound Agrin is localized to caveolin-1- and flotillin-1-enriched lipid raft membranes, while 
a

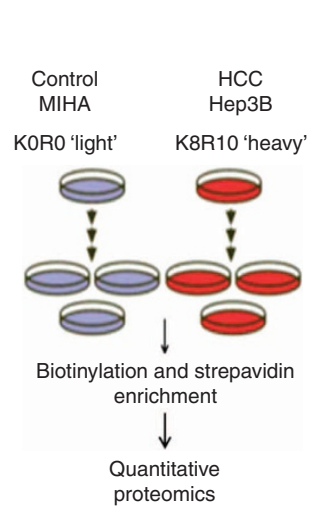

d

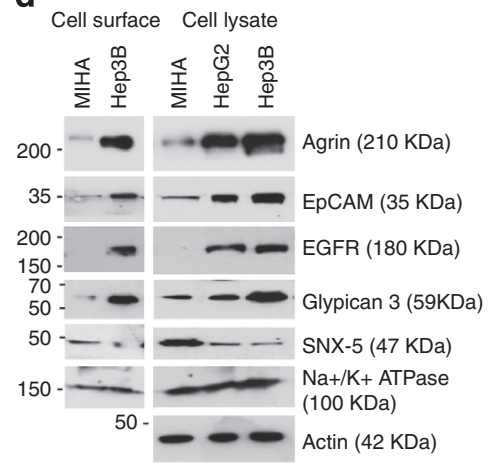

b

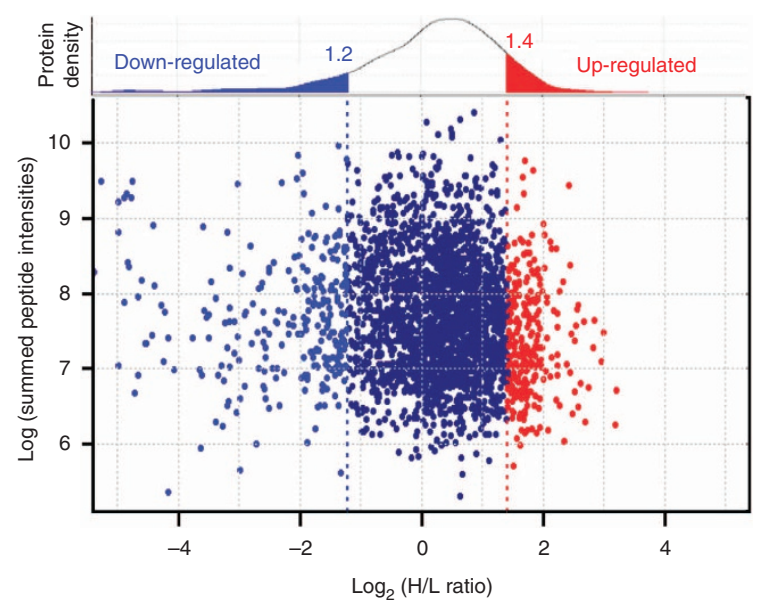

e

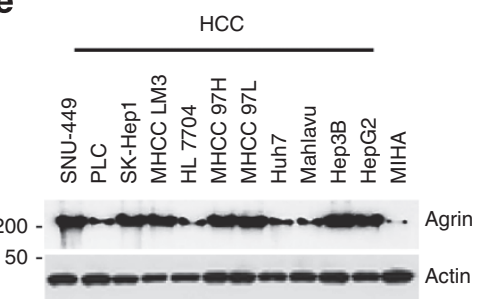

C

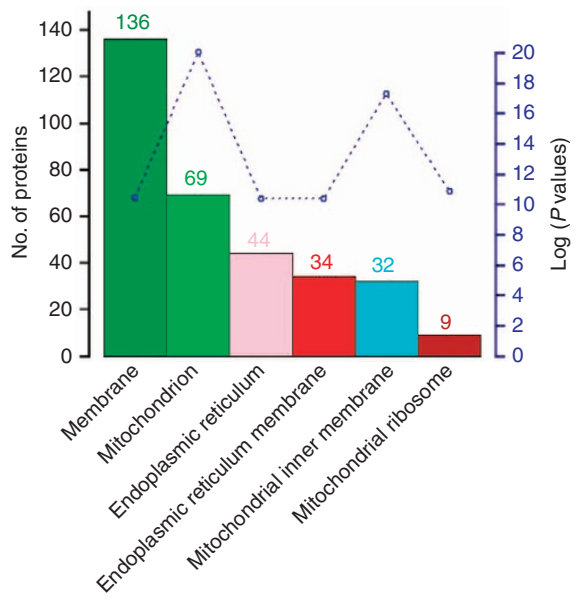

f

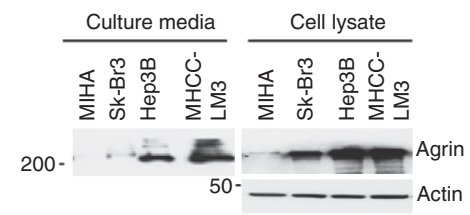

g

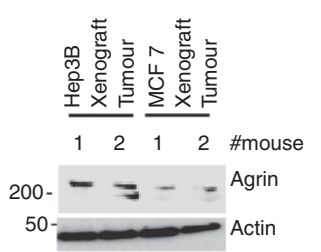

Figure 1 | Identification of Agrin as an overexpressed cell surface protein in HCC cell lines. (a) Schematic workflow of SILAC mass spectrometry-based analysis of cell surface proteins, where MIHA and Hep3B cells are labelled with 'light' and 'heavy' amino acids, respectively, and vice-versa. (b) The ratio intensity plot representing protein fold change (SILAC ratio versus corresponding summed peptide intensity distribution) and protein density plot (upper panel). Red, blue and navy-blue cluster indicates up-, down- and unregulated proteins. (c) Gene ontology Cellular Component analysis of upregulated proteins in Hep3B cells. The scale bar ( $y$ axis right hand side) indicates the $P$ values, while the numbers on top of each bar denotes the abundance of identified proteins in the particular cellular component. (d) Biotinylated cell surface proteins and total cell lysates from the indicated cell lines were analysed by western blot analysis using the indicated primary antibodies. (e) Western blot analysis of Agrin expression in non-tumorigenic MIHA and various HCC cell lines. $\beta$-Actin was used as loading control. (f) Conditioned medium from serum-starved (12 h) cell lines were concentrated and precipitated with trichloroacetic acid and analysed by western blot for secreted Agrin. Total cell lysates from the same cell lines were also probed for Agrin with $\beta$-actin as a loading control. (g) Levels of Agrin in mouse xenografts of indicated cell lines were analysed by western blot using an Agrin-specific monoclonal antibody. $\beta$-Actin served as loading control. $\mathrm{H} / \mathrm{L}$, heavy/light.

a subpopulation of it was associated with endosomal and/or high-density fractions marked by Rab5 and CD-71, respectively (Supplementary Fig. 2a). Analysis of membrane and soluble fractions also revealed robust Agrin levels in Hep3B compared with MIHA cells (Supplementary Fig. 2b). The soluble Agrin may represent those loosely associated with endosomal membranes and/or secreted. To test constitutive Agrin internalization, an Agrin antibody internalization assay was performed. At $4{ }^{\circ} \mathrm{C}$, Agrin antibody at cell surfaces was colocalized with cholera toxinB (CTxB), which binds monosialogangliosides in lipid raft membranes (Supplementary Fig. 2c,d, first panel). After $5 \mathrm{~min}$ incubation at $37^{\circ} \mathrm{C}$, Agrin antibody was co-internalized with $\mathrm{CTxB}$ coupled with coherent signal intensity overlaps projected towards intracellular compartments (Supplementary Fig. 2c,d, second panel) and were in internal organellar compartments by 30 and 40 min (Supplementary Fig. 2c,d, third and fourth panels, respectively). Similarly, internalized Agrin antibody was observed in EEA-1-positive early endosomes from 5 min till the observed 30 min (Supplementary Fig. 2e,f), suggesting that secreted and cell surface Agrin is dynamically endocytosed, consistent with its identification by surface biotinylation and SILAC as a surfaceenriched protein in HCC cells.

Critical role of Agrin in cellular proliferation. To characterize the functional role of Agrin in HCC, we depleted Agrin either by stably transducing with Agrin shRNA-expressing lentiviruses or transfecting a short interfering RNA (siRNA) pool targeting Agrin in highly metastatic MHCC-LM3 and Hep3B cells. Like Hep3B cells, cell surface expression of Agrin in MHCC-LM3 cells was significantly higher than MIHA cells (Supplementary Fig. 3). Agrin knockdown was very efficient in HCC cell lines (Fig. 2a and Supplementary Fig. 4a). Compared with the control cells, Agrin depletion significantly reduced cellular proliferation rates by $42 \%$ (Fig. 2b and Supplementary Fig. 4a) and converted the elongated control cells to a cobblestone shape with enhanced cell apposition, similar to MIHA cells (Fig. 2c and Supplementary Fig. 4b). This morphological change was substantially reversed by addition of soluble recombinant Agrin (Fig. 2c). In addition, MIHA cells 
a

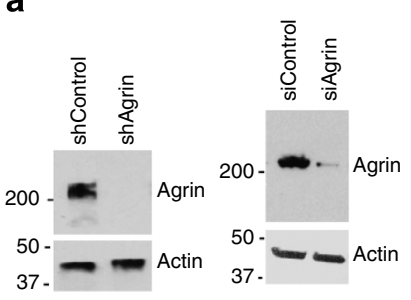

b

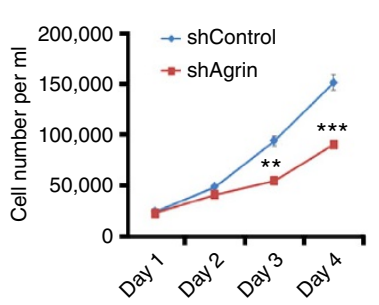

C

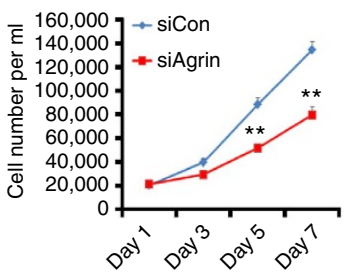

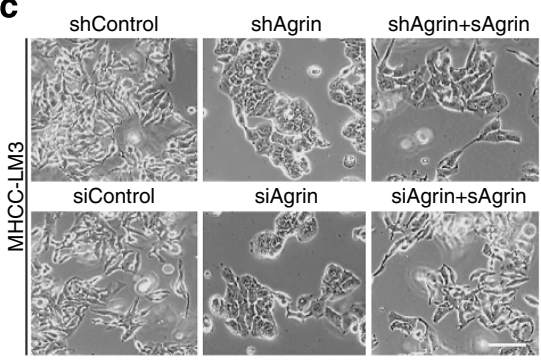

d

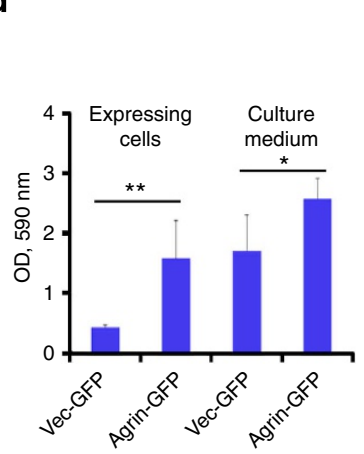

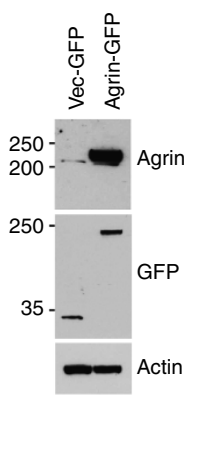
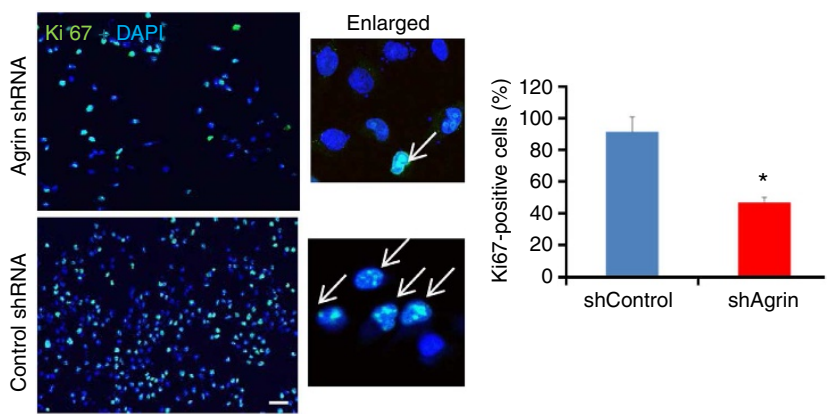

g
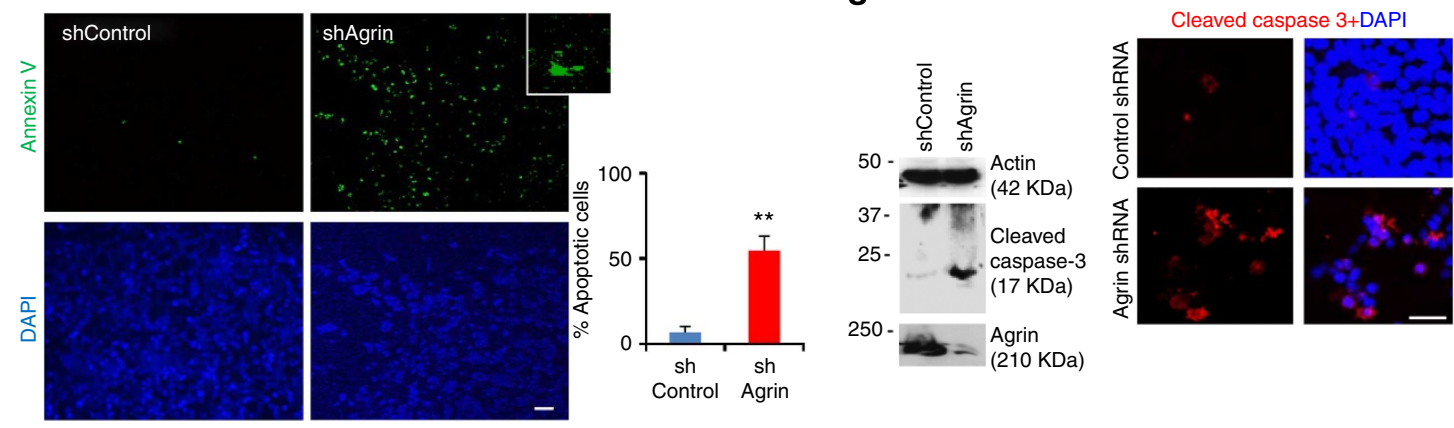

Figure 2 | Agrin knockdown affects cell growth and apoptosis. (a) Western blot in MHCC-LM3 cells transduced with control and shAgrin expressing lentiviruses or transfected with control or siRNA against Agrin. $\beta$-Actin was used as a loading control. (b) Cellular proliferation of control and Agrin knockdown MHCC-LM3 cells when passaged in the indicated days. Error bars represent s.d. of means of three independent experiments performed in triplicates ( ${ }^{\star \star} P$ value $=0.002$ and ${ }^{\star \star \star} P=0.0004$, respectively, Students $t$-test). (c) Bright-field microscopy images of control and Agrin-depleted MHCCLM3 cells either untreated or treated with soluble recombinant Agrin ( $20 \mu \mathrm{g} \mathrm{ml}{ }^{-1}$ ) for 1 day. Scale bar, $10 \mu \mathrm{m}$ (d) MIHA cells expressing either control or Agrin-GFP were subjected to cell proliferation assay after $72 \mathrm{~h}$ post transfection (first two bars). Conditioned media from the above transfected cells were incubated with naive MIHA cells for 1 day and subsequently analysed for cell proliferation assay (third and fourth bars). Error bars represent s.d. of means for three independent experiments performed in triplicates ( ${ }^{\star} P$ value $=0.003,{ }^{\star} P$ value $=0.02$, Student's $t$-test). Western blot analysis of Agrin, GFP expression and $\beta$-actin (as loading control) are shown. (e) Control shRNA and shAgrin-transduced MHCC-LM3 cells were immunostained with Ki67 and counterstained with DAPI. Representative confocal images are shown. Arrows in the enlarged panel indicate actively proliferating cell nuclei. Scale bar, $10 \mu \mathrm{m}$. Quantitative plot depicting the percentage Ki67-positive cells in at least five different microscopic fields and error bars represent s.d. of means ( ${ }^{\star} P$ value $=0.01$, Student's $t$-test). (f) shControl or shAgrin MHCC-LM3 cells were analysed using Annexin $V$ apoptosis staining kit. Representative images co-stained with DAPI are shown and enlarged area inset depicts a typical Annexin V-stained apoptotic cell. Quantitative plot depicting the percentage of apoptotic cells in at least five different microscopic fields is shown. Scale bar, $10 \mu \mathrm{m}$. Error bar represents $\mathrm{s} . \mathrm{d}$. ( ${ }^{\star} P$ value $=0.005$, Student's $t$-test). (g) Western blot analysis of cleaved caspase-3 in shControl and shAgrin MHCC-LM3 cells. The blots were stripped and probed for Agrin and $\beta$-actin. Immunofluorescence analysis for cleaved caspase-3 (red) and DAPI (blue) in control and Agrin shRNA-transduced MHCC-LM3 cells. Scale bar, $10 \mu$ m.

overexpressing Agrin-green fluorescent protein (GFP) showed enhanced proliferation than those expressing vector alone (Fig. 2d). Interestingly, culture media from Agrin overexpressing cells also promoted growth when incubated with naive MIHA cells (Fig. 2d), supporting the notion that secreted Agrin is the functional form. Significant reduction $(\sim 41 \%)$ of proliferation marker Ki67 labelling in Agrin knockdown cells is consistent with reduced proliferation observed upon Agrin depletion in HCC cells (Fig. 2e and Supplementary Fig. 4c).
We next examined whether growth arrest due to Agrin depletion is also associated with increased apoptosis in addition to decreased proliferation. Compared with control cells, we observed $>50 \%$ apoptosis in Agrin knockdown cells, as indicated by Annexin V staining (Fig. 2f). Similarly cleaved caspase-3 (a late-phase apoptosis marker) was elevated in Agrin-depleted cells (Fig. 2g and Supplementary Fig. 4d). Hence, suppressing Agrin expression reduced cell proliferation and induced apoptosis in HCC cell lines. 
Agrin functions in cellular motility and invasion. We next investigated whether morphological and proliferative changes in Agrin-depleted cells affected migratory and invasive behaviour. Agrin depletion severely reduced the migration of MHCC-LM3 cells in a wound-healing assay (Fig. 3a). While shControl cells almost recovered the wound by $48 \mathrm{~h}$, shAgrin cells had significant wound area unhealed (Fig. 3a). Interestingly, soluble recombinant Agrin significantly rescued the migration of Agrin-depleted cells $30 \mathrm{~h}$ post-wound scratch (Fig. 3b). Agrin-depleted cells also exhibited significantly lower ( $>50 \%$ reduction) infiltration rates in transwell migration and invasion assays than control cells (Fig. 3c,d and Supplementary Fig. 4e). Again, these migration and invasion defects in knockdown cells were significantly rescued either by supplementation of soluble Agrin (Fig. 3c) or expressing full-length rat Agrin-GFP (Fig. 3d). These results cumulatively suggest that the observed phenotype in Agrin knockdown cells is specifically attributed to reduced Agrin levels. To further demonstrate a role of Agrin in liver tumorigenesis, anchorageindependent growth of control and Agrin knockdown cells was assessed. As expected, Agrin-depleted cells significantly reduced the colony formation by $\sim 65 \%$ compared with control cells (Fig. 3e). Compared with well-developed colonies observed in control cells, shAgrin cell colonies were fewer and relatively smaller in size (Fig. 3e, enlarged panel). Therefore, these results suggest that Agrin is critical for oncogenic property of liver cancer cells.

Agrin controls membrane ruffling and invadopodia. Loss of cellular invasion upon Agrin depletion associated with altered

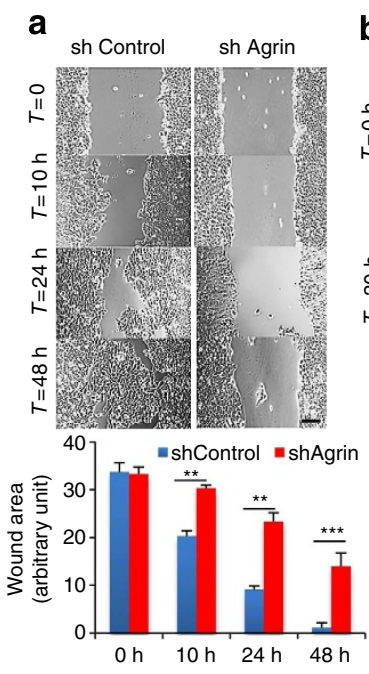

b
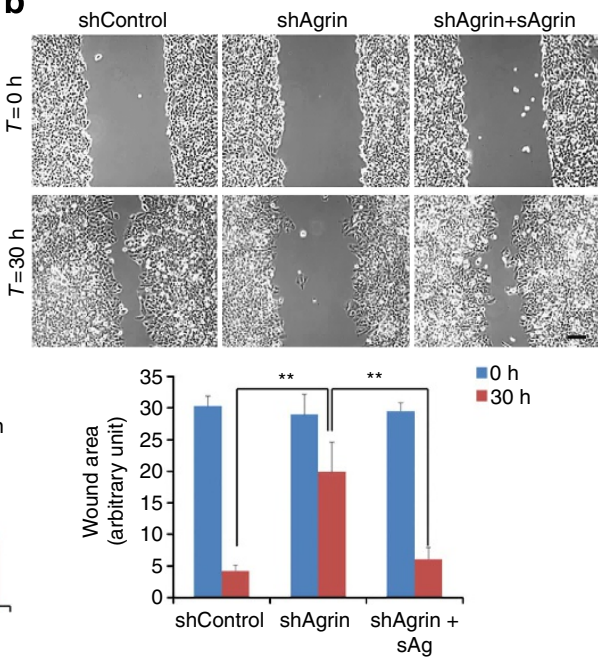

$=0 \mathrm{~h}$

$30 \mathrm{~h}$

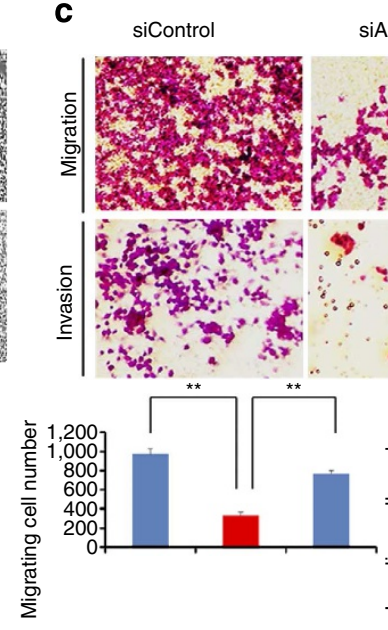

siAgrin

siAgrin+
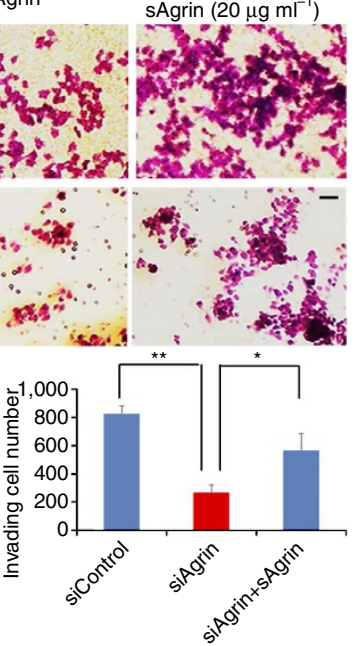

d

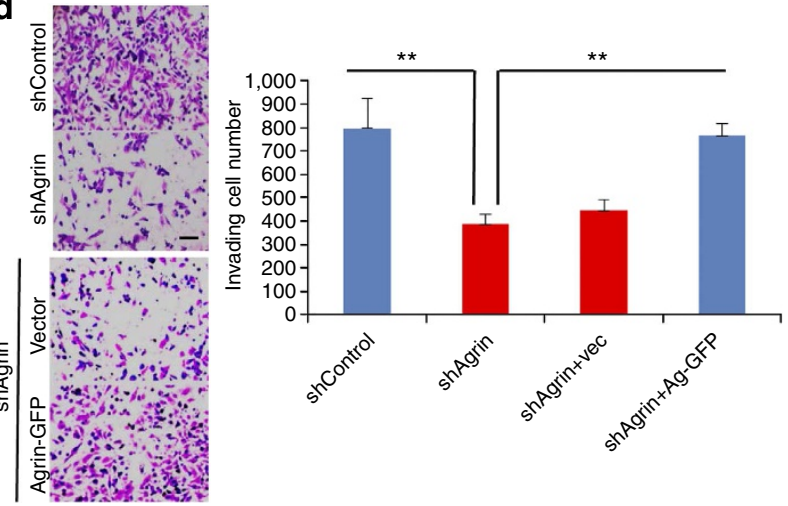

e
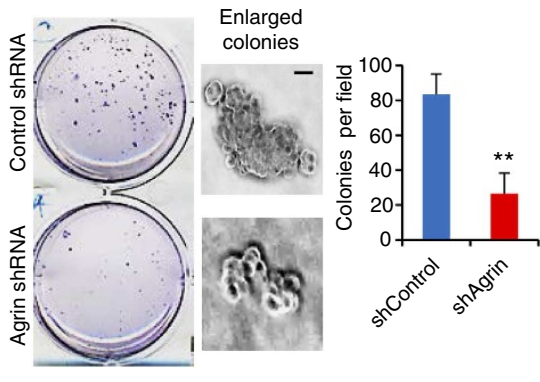

Figure 3 | Agrin regulates cancer cell migration, invasion and colony formation. (a) Wound-healing assay performed in shControl or shAgrin MHCCLM3 cells. Representative images are shown at indicated time points and results are quantified using Image software (magnification $\times 20$ ). The area left unhealed was measured by ImageJ software in arbitrary units and error bars represent s.d for three independent experiments performed in triplicates $\left({ }^{\star \star} P\right.$ value $=0.003,{ }^{\star \star \star} P$ value $<0.0003$, respectively, Student's $t$-test). Scale bars, $50 \mu m$. (b) Wound-healing assay performed in control, Agrin depleted

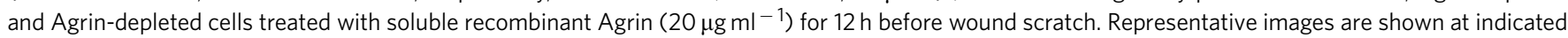
time points and results are quantified as in a. Error bars represent s.d. ( ${ }^{\star \star} P$ value $<0.005$, Student's $t$-test). Scale bar, $50 \mu m$. (c) Control or Agrin knockdown MHCC-LM3 cells were either untreated or treated with soluble recombinant Agrin $\left(20 \mu \mathrm{g} \mathrm{ml}{ }^{-1}\right)$ for $12 \mathrm{~h}$ and subjected to transwell migration (without Matrigel) and invasion (with Matrigel) assays. Migratory or invasive cells are imaged in a bright-field microscope under $\times 20$ magnification. Results are also quantified using ImageJ software and error bars represent s.d. of means for three independent experiments performed in triplicates $\left({ }^{\star \star} P\right.$ value $=0.001,{ }^{\star} P$ value $=0.02$, respectively, Student's $t$-test). Scale bar, $50 \mu \mathrm{m}$. (d) Control or Agrin knockdown MHCC-LM3 cells either expressing GFP-vector or Agrin-GFP were subjected to a Matrigel invasion assay. Cells invading Matrigel were imaged in a bright-field microscope under $\times 20$ magnification. Results were also quantified as in c. Error bars indicate s.d. of means of three independent biological replicates performed in triplicates ( ${ }^{\star \star} P$ values $=0.005$ and 0.001 , respectively, Student's $t$-test). Scale bar, $50 \mu \mathrm{m}$. (e) Control or Agrin knockdown MHCC-LM3 cells were subjected to a soft agar clonogenic assay. Representative pictures for colony growth are shown. Typical cell colonies observed by bright-field microscopy at day 10 of growth in soft agar is also shown. Quantification of the number of colonies is shown. Error bars indicate s.d of mean of three independent experiments performed in triplicates ( ${ }^{\star \star} P$ value $=0.004$, Student's $t$-test). Scale bars, $50 \mu \mathrm{m}$. 
a

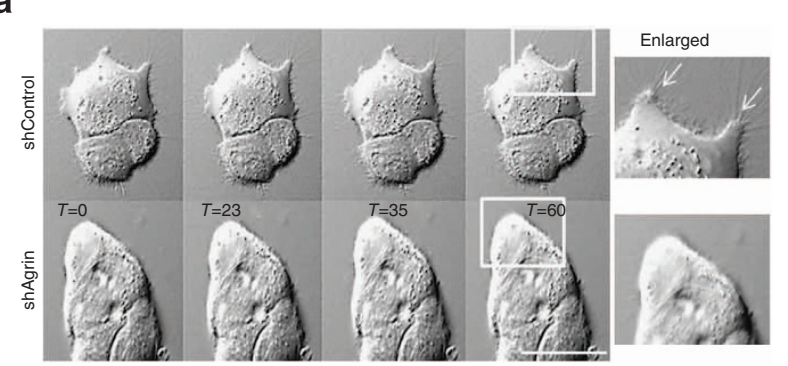

b

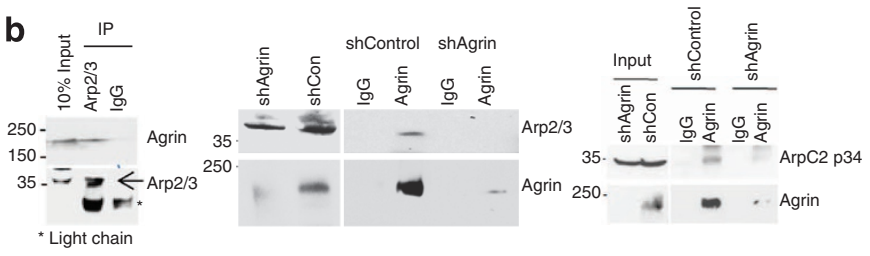

c

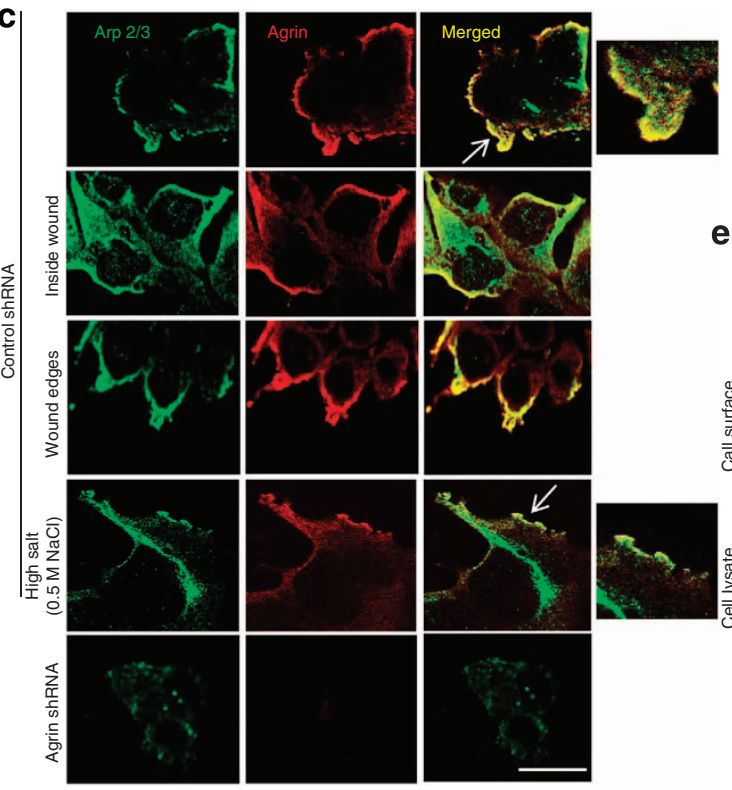

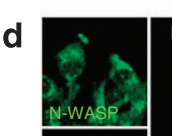
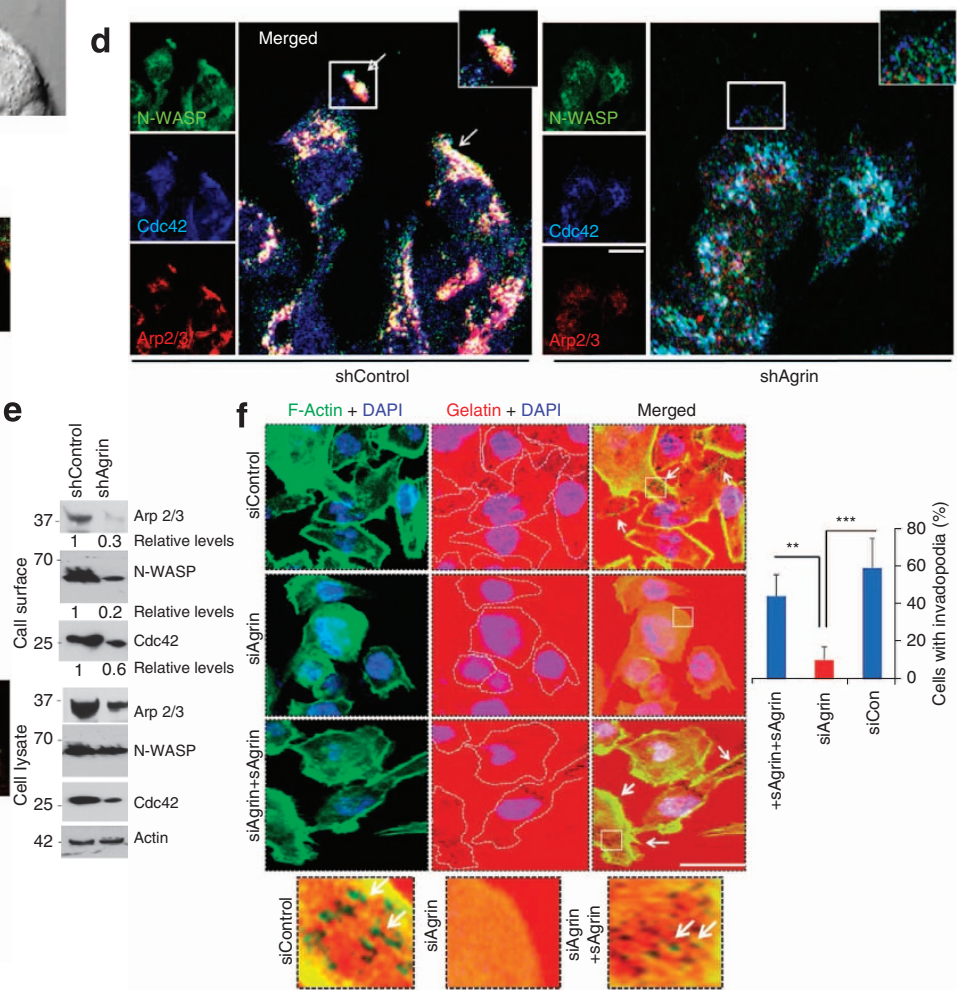

Figure 4 | Agrin regulates ruffling and invadopodia in HCC cell lines. (a) Representative live-cell time-lapse differential interference contrast images of control and Agrin knockdown cells at indicated time frames (in seconds) are shown. Scale bar, $10 \mu \mathrm{m}$. Boxed area is represented as enlarged panels. Arrows indicate membrane ruffles/protrusions. (b) MHCC-LM3 cells IP using Arp2/3 subunit 1B (left panel) or Agrin antibodies (middle and rightmost panels) and IgG were analysed by western blotting for Agrin, Arp2/3 subunits 1B or ArpC2p34. The blot was stripped and re-probed for Arp2/3 or Agrin. Thirty micrograms (10\%) of whole-cell lysate were used as input control. Lysates from Agrin knockdown cells (right panel) were used as negative control. (c) Immunofluorescence analysis of control and Agrin knockdown MHCC-LM3 cells $12 \mathrm{~h}$ post scratch assay using goat Arp2/3 antibody and mouse Agrin antibody. Representative confocal images are shown. Panels (ii and iii) represent images of cells within the wound area and edges of wound, respectively. For panel (iv), cells were washed two times with high salt wash buffer $(0.5 \mathrm{M} \mathrm{NaCl})$ for $10 \mathrm{~min}$ at RT before fixation and immunostaining. Enlarged $Z$ sections were performed at the leading edge of membrane ruffles marked by white arrows. Scale bar, $10 \mu \mathrm{m}$. (d) Cells were processed similarly as in $\mathbf{c}$ and immunostained with mouse anti-WASP, rabbit anti-Cdc42 and goat anti-Arp2/3. Boxed area is represented as enlarged panel inset. Scale bar, $10 \mu \mathrm{m}$.

(e) Biotinylated surface proteins from control or Agrin knockdown MHCC-LM3 cells were analysed by a western blot for indicated proteins. Total cell lysate was also western blotted and $\beta$-actin served as a loading control. (f) Control and Agrin siRNA-transfected cells either untreated or treated with soluble Agrin $\left(20 \mu \mathrm{g} \mathrm{ml}^{-1}\right)$ for $12 \mathrm{~h}$ were cultured on Cy3-Gelatin for another $12 \mathrm{~h}$, fixed and then stained with F-actin and DAPI. Representative confocal images are shown and invadopodia was quantified using ImageJ. Boxed areas are represented as enlarged lower panels. Arrows indicate F-actin-enriched degraded gelatin areas. Scale bar, $10 \mu \mathrm{m}$. At least five different fields were quantified and error bars indicate the s.d. of means of at least three independent experiments ( ${ }^{\star \star \star} P$ value $=0.0005,{ }^{\star \star} P$ value $=0.004$, respectively, Student's $t$ test).

membrane ruffling and/or invadopodia are hallmarks of invasive cancer cells ${ }^{29,30}$. Dorsal ruffles and protrusions observed in control cells were markedly reduced upon Agrin depletion (Fig. 4a, and Supplementary Movies 1 and 2). Since these invasive structures are largely governed by association of actinrelated Arp2/3, N-WASP and Cdc42 complex near cell surface ${ }^{31}$, we deciphered whether Agrin is involved in associating Arp2/3 subunits to the inner leaflet of cell membrane to regulate ruffling. Co-immunoprecipitation (co-IP) with either Arp2/3 (subunits 1B and ArpC2p34) or Agrin antibody revealed a specific interaction of Agrin with Arp2/3 complex (Fig. 4b). As Arp2/3 and Agrin are distributed on the inside and outside of the plasma membrane, we interpret their co-IP as indirect interaction most likely mediated by transmembrane protein complexes. Agrin and Arp2/3 subunit
$1 \mathrm{~B}$ colocalized at the leading edge of membrane ruffles in a group of migratory cells $12 \mathrm{~h}$ post a wound scratch assay (Fig. 4c, panel i). Agrin similarly colocalized with Arp2/3 within dense cells and towards the edge of the wound, ruling out the possibility of cell density or wound stress mediated colocalization between the two proteins (Fig. 4c, panels ii,iii). High salt wash that removes peripherally associated proteins, partially affected the colocalization at the leading edge of membrane ruffles (Fig. 4c, panel iv). In contrast, Agrin-depleted cells had greatly reduced Arp $2 / 3$ at leading edges (Fig. 4c, panel v). Further, Arp2/3, $\mathrm{N}$-WASP and Cdc42 colocalized at leading edges of migrating control cells that were greatly hampered by Agrin depletion (Fig. $4 d)$. In addition, association with the inner leaflet of cell membrane and total protein levels of Arp $2 / 3, \mathrm{~N}-\mathrm{WASP}$ and 
Cdc42 were also reduced by $70 \%, 80 \%$ and $40 \%$, respectively, upon Agrin depletion (Fig. 4e).

As invadopodia are distinct from membrane ruffles in being protrusive matrix-degrading structures on the ventral surface of cancer cells enriched with F-actin and cortactin in addition to the above-mentioned markers ${ }^{32,33}$, we examined Agrin's role in invadopodia formation by a gelatin-invadopodia assay simulating degradation of ECM. F-Actin-enriched invadopodia visualized by gelatin-degrading activity were abundant on the ventral surfaces of control cells (Fig. 4f, top panels). Significantly, invadopodia were greatly reduced in Agrin-depleted cells (Fig. 4f, middle panels). Addition of soluble Agrin restored the invadopodia formation in knockdown cells (Fig. 4f, bottom panels). Moreover, Agrin-cortactin clusters that localized to invadopodia and degraded matrix sites were diminished upon Agrin depletion (Supplementary Fig. 5a,b). Together, these results suggest that Agrin governs Arp2/3 complex-dependent ruffling and invadopodia formation in HCC cells.

Agrin affects EMT and integrin-FA signalling. Enhanced cohesiveness of Agrin-depleted cells, in the context that vimentin is one of the proteins enriched in HCC cancer cells that was recovered in our SILAC analysis likely due to interaction with other surface proteins (Supplementary Table 1), suggests that Agrin may be involved in maintaining mesenchymal property of HCC cells. Consistently, along with reduced mRNA levels of Arp2/3, reduction of vimentin mRNA was also observed in Agrin-depleted cells (Supplementary Fig. 6a). Therefore, we tested the hypothesis that Agrin might have a role in vimentin's association with plasma membrane. As shown, co-IP with an antibody against vimentin co-recovered Agrin from Hep3B cells but not in MIHA cells (Supplementary Fig. 6b). Agrin depletion increased epithelial marker E-cadherin by 2.7 folds but reduced mesenchymal markers $\mathrm{N}$-cadherin, vimentin and Snail- 1 by $37 \%$, $44 \%$ and $30 \%$, respectively (Fig. 5a). Elevated E-cadherin levels coupled with reduced vimentin in Agrin-depleted cells was reversed by addition of soluble Agrin (Supplementary Fig. $6 \mathrm{c}, \mathrm{d}$ ), suggesting that secreted Agrin alters EMT markers and regulates their association with inner cell membrane (Supplementary Fig. 6e). Similar results were also observed upon siRNAmediated Agrin depletion with no effect on glypican-3 (Supplementary Fig. 6f). These lines of evidence support a role of Agrin in maintaining mesenchymal characteristics of HCC cells.

Mechanistic investigation of Agrin's role in modulating the integrin-FAK pathway instrumental in recruiting mesenchymal markers to cell surface and regulating membrane protrusions ${ }^{34,35}$ revealed that Agrin depletion markedly reduced FA proteins integrin $\beta 1$ and pY397 FAK by $85 \%$ and $88 \%$, respectively (Fig. 5b). Addition of soluble Agrin restored pY397 FAK level (Supplementary Fig. 6d). In contrast, Agrin depletion had lesser inhibition on pSrc and pPI3-K signalling (Fig. 5b). Notably, Agrin knockdown also reduced pAkt and pErk1/2 signalling by $87 \%$ and $61 \%$, respectively (Fig. 5b and Supplementary Fig. 6f), likely accounting for reduced cell proliferation. Furthermore, an interaction between Agrin and integrin $\beta 1$ was observed (Fig. $5 \mathrm{c}$ upper panel and Supplementary Fig. 7a). Agrin was coIP with activated (pY397) and total FAK (Fig. 5c middle and lower panels, and Supplementary Fig. 7b). In addition to significant colocalization observed without stimulation, activation of integrin and FAs by $\mathrm{Mn}^{+2}$ led to an increased Agrin clustering in FAs (Fig. 5d, panels i and ii). In contrast, integrin $\beta 1$ appeared dispersed and not restricted to FA in Agrindepleted cells (Fig. 5d, panel iii). Even $\mathrm{Mn}^{+2}$ stimulation in these cells failed to activate the integrin-adhesion complexes (Fig. 5d, panel iv), suggesting that Agrin associates with FAs and its depletion affects FA integrity.
Agrin as ECM sensor regulating FAs and cell attachment. To address how Agrin provides ECM cues to regulate FAs, we first examined its effect on FA integrity. Control cells exhibited Agrin and phospho-Focal adhesion kinase (pFAK) (pY397) colocalization while Agrin-depleted cells had reduced $\mathrm{pFAK}$ and FA lengths similar to disrupted FAs induced by myosin IIA bleb inhibitor blebbistatin $^{35}$ (Fig. 5e and Supplementary Fig. 8a). In elongated appearing control cells, pFAK colocalized with other FA components p-Paxillin (Tyr118) and vinculin at activated FAs (Supplementary Fig. 8b,c, left panels i). Agrin-depleted cells were circular and widespread with disrupted FAs (Supplementary Fig. 8b,c, left panels ii). Since defect in cell morphology correlates with poor FA alignment and loss of invasiveness ${ }^{36}$, we further investigated whether Agrin depletion induced FA disruption affected cell attachment. Immediately, post plating on fibronectin-coated dishes, control and Agrin knockdown cells appeared rounded (Fig. 5f, $T=0 \mathrm{~h}$ panel). After $2 \mathrm{~h}$, control cells started to elongate, whereas Agrin-depleted cells remained circular (Fig. $5 \mathrm{f}, T=2 \mathrm{~h}$ panel). At $4 \mathrm{~h}$ post plating, both control and Agrin-depleted cells attached; the former typically elongated with aligned FA activity, whereas the latter widespread with poor FA activity (Fig. 5f, $T=4 \mathrm{~h}$ panel and Supplementary Fig. 8d). The cell elongation ratio was 3.53 and $1.54 \mu \mathrm{m}$ for control and Agrin knockdown cells, respectively (Supplementary Fig. 8d). Furthermore, at $3 \mathrm{~h}$ post plating, control cells expressing Paxillin-GFP had well-defined FAs that appeared fragmented upon Agrin depletion (Fig. 5g, and Supplementary). This FA defect was substantially rescued by exogenous Agrin treatment (Fig. 5g and Supplementary Movie 5), confirming the specific role of Agrin in controlling FA integrity.

Agrin promotes FAK dependant ECM degradation. Since the defined pFAK-cortactin cluster length, colocalization in migrating cells $16 \mathrm{~h}$ post scratch assay and association as analysed by co-IP were hampered upon Agrin depletion (Supplementary Fig. 9a,b), we explored whether Agrin, FAK or their dual involvement regulates invadopodia formation and matrix degradation. Consistently, some activated FA components (pY397FAK and p-Paxillin) localized near or within areas of degraded matrix in control cells as reported before ${ }^{37,38}$ (Supplementary Fig. 9c-e). Interestingly, Agrin depletion affected FA activity and prevented matrix degradation (Supplementary Fig. 9c-e). Although FAK knockdown reduced invadopodia and matrix degradation by $\sim 50 \%$, Agrin and FAK double depletion had significantly greater inhibition (up to 82\%; Supplementary Fig. 10a,b). Moreover, MIHA cells with low endogenous Agrin levels had negligible invadopodia and matrix degradation despite the presence of pFAK (Supplementary Fig. 10c). Interestingly, supplementing Agrin increased invadopodia by $\sim 50 \%$ with pFAK being observed within some degraded areas. These matrix degradation were substantially reduced in FAK-depleted cells treated with Agrin (Supplementary Fig. 10c), suggesting that Agrin functions through FAK-dependent ECM degradation.

Cumulative impact of FAs and Agrin on tumour cell growth. The role of FAK in promoting tumour growth is reported in various cancers such that FAK inhibitors have been proposed as anti-tumorigenic agents ${ }^{24-27}$. To establish that Agrin-mediated regulation of FAs affects tumour cell growth, we used FAK inhibitor PF-562271 that reduced pFAK activity and spheroid culture diameter in a dose-responsive manner (Supplementary Fig. 11a,b). Although inhibitory effects of $0.8 \mu \mathrm{M}$ FAK inhibitor alone in spheroid and soft agar growth were slightly lesser than that of Agrin depletion, PF-562271 treatment in Agrin-depleted cells cumulatively had significantly greater anti-growth effects 
a

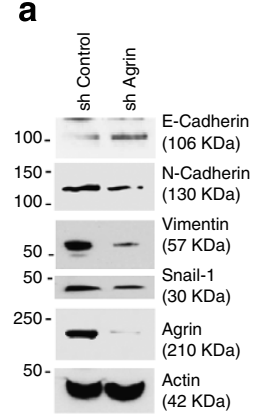

b

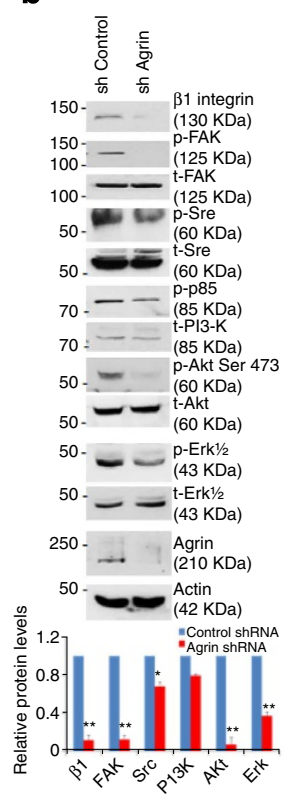

C

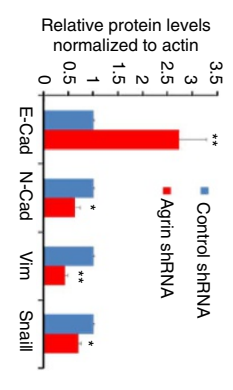

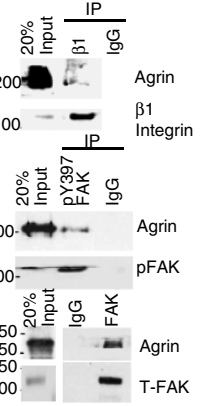

d

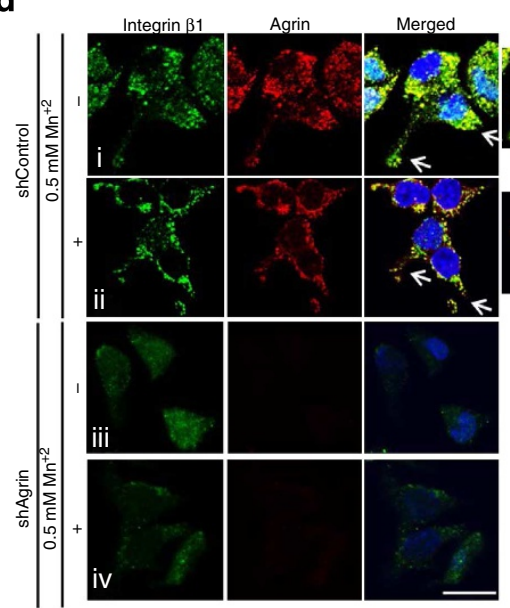

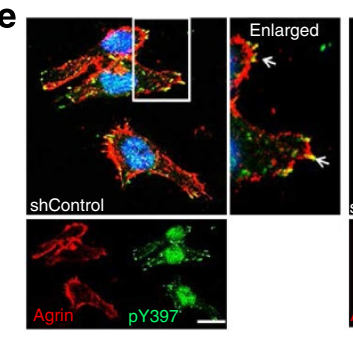
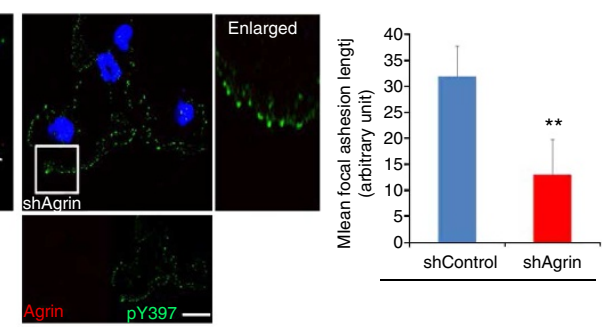

f

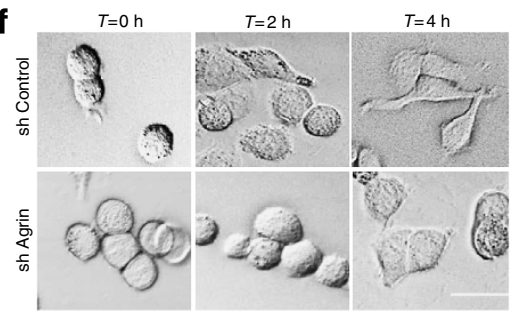
XZ-Stack
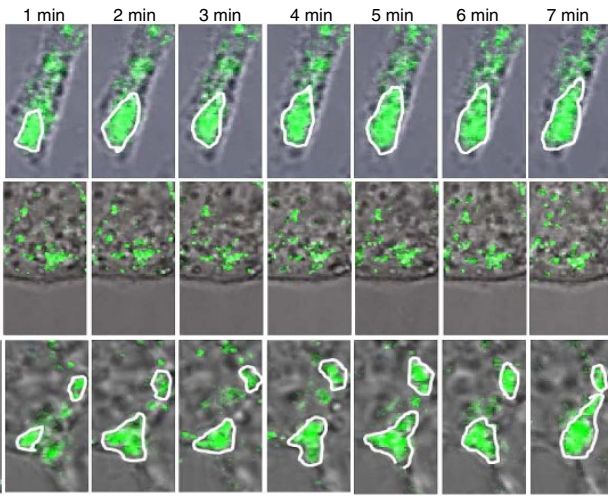

Figure 5 | Agrin as an ECM sensor for EMT and FA integrity. (a,b) Total lysates of control or Agrin shRNA MHCC-LM3-transduced cells were analysed by western blots using antibodies against the indicated proteins. Quantification of proteins normalized to actin (a) or respective total protein levels (b) are depicted graphically. Error bars represent s.d. ( ${ }^{\star} P$ value $<0.04,{ }^{\star \star} P$ value $<0.002$, Student's $t$-test). (c) MHCC-LM3 cell lysates were IP with either rabbit IgG or anti-integrin $\beta 1$ (upper panel), anti-FAK pY397 (middle panel) or anti-total FAK (T-FAK) antibody (lower panel) and western blotted for Agrin. The blot was stripped and re-probed for integrin $\beta 1$ (upper panel), pFAK (middle panel) or T-FAK (lower panel). Sixty micrograms (20\%) of whole-cell lysate were used as input control. (d) Control or Agrin-depleted cells were either mock or pre-treated with $0.5 \mathrm{mM} \mathrm{MnCl}_{2}$ solution in complete media for 30 min at $37^{\circ} \mathrm{C}$ to activate $\mathrm{FAs}$, washed and processed for immunofluorescence using rabbit integrin $\beta 1$ and mouse Agrin antibodies. Representative images co-stained with TO-PRO3 are shown. White arrows indicate the enlarged area of cells where $Z$ section was performed. Scale bar, $10 \mu \mathrm{m}$. (e) Control or Agrin-depleted MHCC-LM3 cells were immunostained using mouse anti-Agrin and rabbit anti-FAK (pY397) antibodies. Representative confocal images co-stained with TO-PRO3 are shown. Arrows indicate colocalization and boxed areas are represented as enlarged panels. FA length was analysed by ImageJ software and at least 10 different fields were chosen for analysis and data represents mean \pm s.d. ( ${ }^{\star} p$ value $=0.005$, Student's $t$-test). Scale bar: $10 \mu$ m. (f) Control or Agrin-depleted MHCC-LM3 cells plated on fibronectin $\left(10 \mu \mathrm{g} \mathrm{ml}^{-1}\right)$-coated dishes. Morphological changes analysed by time-lapse differential interference contrast microscopy live-cell imaging at indicated time points. Scale bar, $10 \mu \mathrm{m}$. (g) Paxillin-GFP expressing control, Agrin-depleted MHCC-LM3 cells alone or supplemented with $10 \mu \mathrm{g} \mathrm{ml}^{-1}$ sAgrin for 1 day were plated on fibronectin-coated dishes. Three hours post plating, cells were imaged live for $7 \mathrm{~min}$ at an interval for $1 \mathrm{~min}$. Representative time-lapse series of boxed region is enlarged and shown. Scale bar, $10 \mu \mathrm{m}$.

(Supplementary Fig. 11b,c), suggesting the importance of Agrin-FAK synergism in in vitro tumorigenesis.

Exogenous Agrin rescues defects of Agrin knockdown cells. We next rationalized that restoring Agrin should rescue the effects caused by Agrin shRNAs if these phenotypes are primarily attributable to reduced levels of Agrin. Hence, we expressed fulllength rat Agrin-GFP (resistant to the shRNA targeting human Agrin) in Agrin knockdown MHCC-LM3 cells, as depicted by Agrin and GFP western blots, respectively (Fig. 6a, first panel and last panel). Compared with vector control, Agrin rescued cells exhibited 7.8 and 3.9 folds increase in activated FAK (pY397) and integrin- $\beta 1$ levels, respectively, while un-transfected control and
Agrin-depleted cells showed reduced pFAK and integrin levels as shown earlier (Fig. 6a). Agrin restoration increased N-cadherin by 2.9 folds and reduced E-cadherin by $48 \%$ (Fig. 6a). Compared with control cells, we also observed reduced FAK activity (by $86 \%$ ) and Arp $2 / 3$ levels (by 93\%) $2 \mathrm{~h}$ post attachment on fibronectin-coated plates upon Agrin depletion (Fig. 6b). Importantly, supplementing Agrin with fibronectin in ECM rescued adhesion defects by increasing pFAK and Arp2/3 levels by $72 \%$ and $55 \%$, respectively (Fig. 6c). Moreover, actin polymerization activity by Arp $2 / 3$ complex reduced by $44 \%$ upon Agrin depletion but was substantially rescued by soluble Agrin (Supplementary Fig. 12). These results cumulatively validate the role of Agrin as an ECM sensor regulating FA and Arp2/3dependent cell attachment. 
a

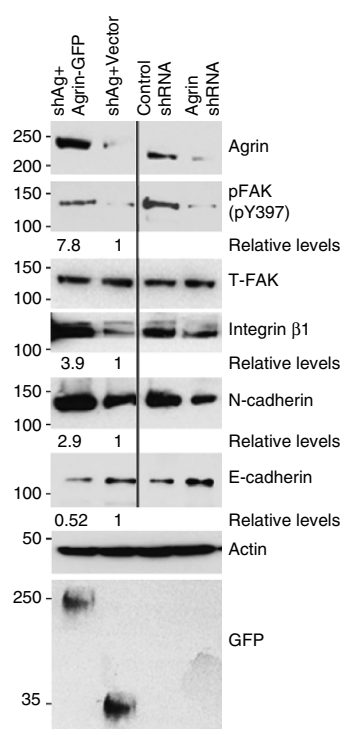

b

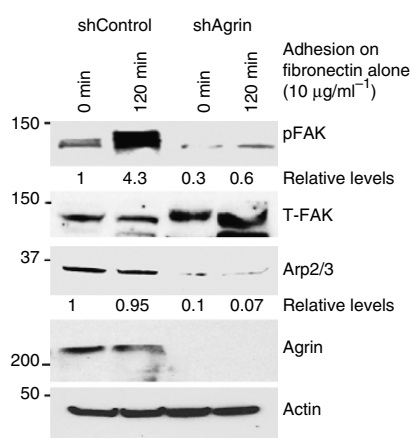

C

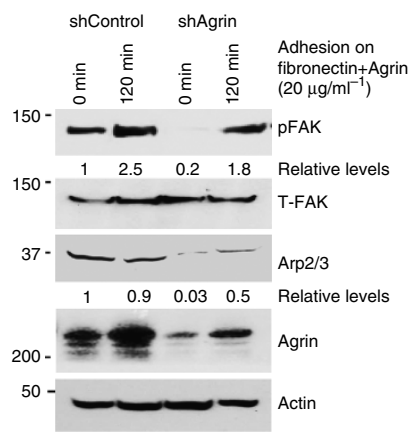

d shAgrin shControl

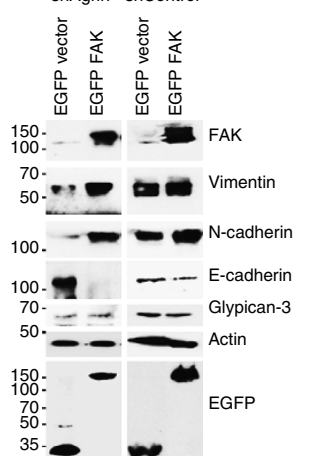

g

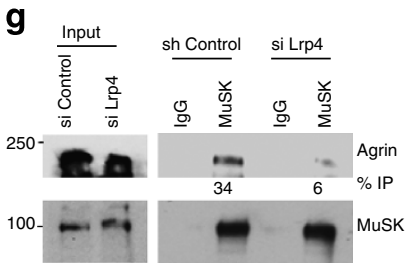

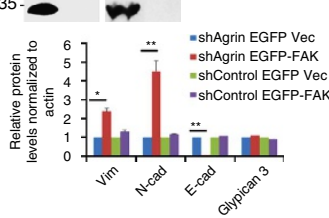

h

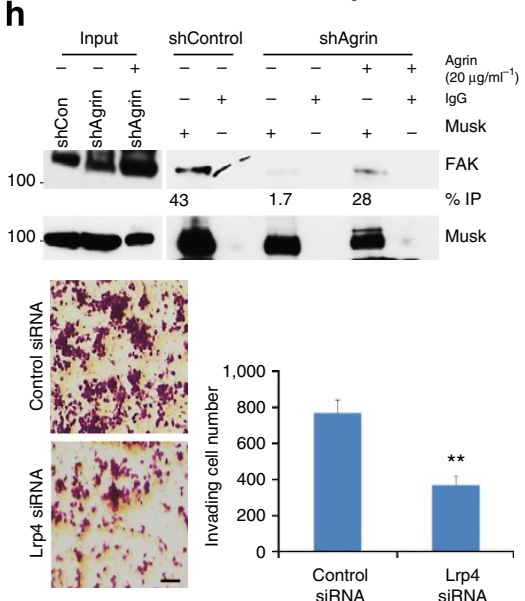

Figure 6 | Oncogenic property of Agrin-Lrp4-MuSK axis is primarily mediated by FAK. (a) Control and Agrin-depleted MHCC-LM3 cells transfected with GFP-vector or full-length Agrin-GFP and total cell lysates were western blotted for indicated proteins. (b,c) Serum-starved control or Agrin-depleted MHCC-LM3 cells were trypsinized and plated on fibronectin alone (b) or fibronectin and $20 \mu \mathrm{gl}^{-1}$ recombinant Agrin (c)-coated surface. Total lysates were analysed by western blots for indicated proteins. $\beta$-Actin served as loading control. (d) Control or Agrin-depleted MHCC-LM3 cells transfected with EGFP-vector or EGFP-FAK and total cell lysates were Western blotted for the indicated proteins. Quantification of proteins normalized to actin is depicted. Error bars represent s.d. ( ${ }^{\star} P$ value $<0.03,{ }^{\star \star} P$ value $<0.002$, Student's $t$-test). (e) Control or Agrin-depleted MHCC-LM3 cells were IP with MuSK antibody and western blotted using tyrosine-specific $4 \mathrm{G} 10$ antibody. The blot was re-probed for MuSK, Agrin and $\beta$-actin (as loading control). (f) Control or Agrin-depleted MHCC-LM3 cells were IP with rabbit MuSK or control IgG antibodies and Western blotted for Lrp4. The blot was stripped and re-probed for MuSK. Thirty micrograms (10\%) cell lysates were used as input controls. ( $(\mathbf{g})$ Control or Lrp4-depleted MHCC-LM3 cells were IP as in $\mathbf{f}$, western blotted for Agrin. The blot was stripped and re-probed as in $\mathbf{f}$. (h) Cells same as in $\mathbf{f}$ were either untreated or treated with $20 \mu \mathrm{g} \mathrm{ml} \mathrm{m}^{-1}$ recombinant Agrin for $12 \mathrm{~h}$ and IP with rabbit IgG or MuSK, and western blotted for FAK. The blot was stripped re-probed for MuSK expression. Thirty micrograms (10\%) of lysate was used as input control. (i) Control or MuSK-depleted cells were IP with FAK antibody and western blotted for Agrin. Thirty micrograms (10\%) of protein lysate was used as input control. The blot was stripped re-probed for FAK expression. Lower panels represent MuSK knockdown and $\beta$-actin as loading control. (j) Control, MuSK and Lrp4 knockdown MHCC-LM3 cells were subjected to a Matrigel invasion assay. Representative images under $\times 10$ magnification are shown and results were quantified using ImageJ software. Error bars represent s.d. ${ }^{\star \star} P$ values $<0.005$ and 0.004 , respectively, Student's $t$-test). Scale bars, $50 \mu \mathrm{m}$.

FAK overexpression suppresses Agrin-depleted phenotype. Recent studies indicate that FAK is critical for HCC progression ${ }^{22}$. If the key function of Agrin is to maintain and sustain FAK activity in HCC cells, then the phenotype caused by Agrin knockdown is primarily due to decreased FAK activity. If this is indeed the case, then we should predict that overexpression of active FAK alone could suppress, at least in part, the phenotype caused by Agrin depletion. Accordingly, EGFP-FAKpY397 expression in Agrindepleted cells restored the mesenchymal markers vimentin and $\mathrm{N}$-cadherin by 2.4 and 4.4 folds, respectively, and decreased E-cadherin by $95 \%$ (Fig. 6d, shAg panel). Respective FAK and enhanced-green fluorescent protein (EGFP) expression levels are also shown (Fig. 6d). However, FAK expression in Agrin knockdown cells did not affect glypican-3, suggesting the specificity of FAK to rescue Agrin-depletion-mediated effects (Fig. 6d). We also did not observe any major effects of FAK overexpression in control shRNA-transduced cells (Fig. 6d, shControl panel). Together, these observations reveal a synergistic dependence of Agrin on FAK activity to drive EMT programme and strongly suggest that FAK is a major mediator of Agrin function.

Agrin-Lrp4-MuSK complex as an oncogenic signalling axis. Since neuronal Agrin containing splice variants within $\mathrm{Z}$ exons preferentially activate Lrp4-MuSK signalling ${ }^{15,16}$, it is important 
a

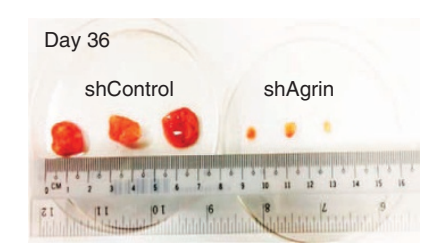

e

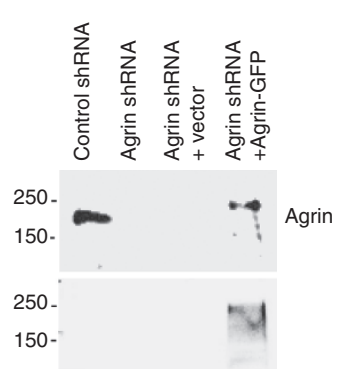

GFP

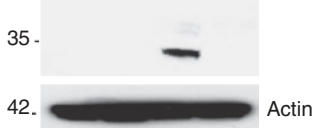

$f$ b
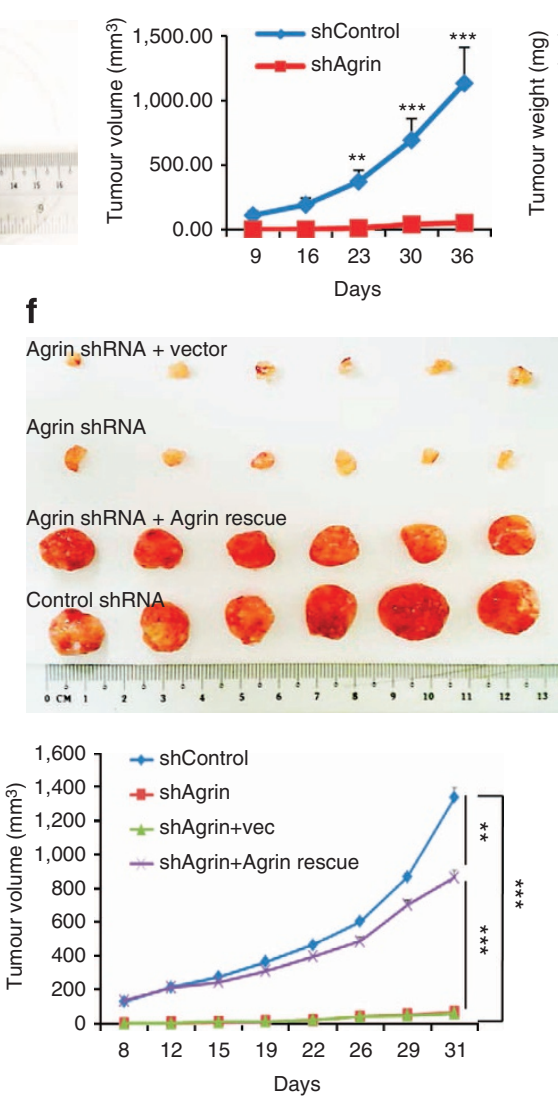

C

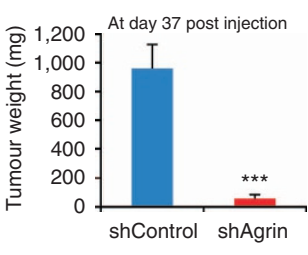

g

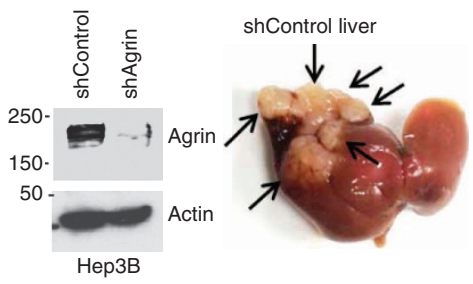

h

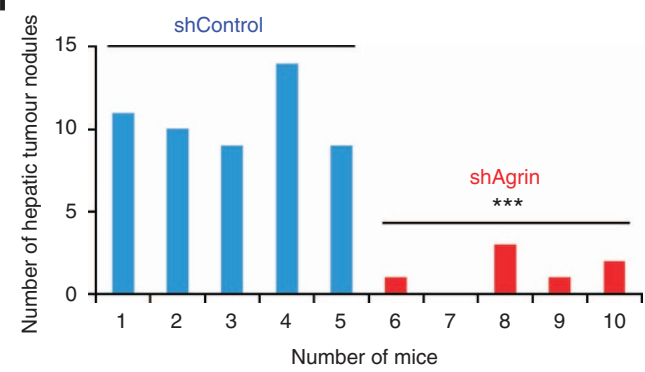

Figure 7 | Agrin regulates oncogenic signalling, tumour growth in xenograft and orthotopic HCC models. (a) Control or Agrin shRNA-expressing MHCC-LM3 cells $\left(1 \times 10^{7}\right.$ cells per $\left.\mathrm{ml}\right)$ were injected subcutaneously in athymic nude mice to establish tumours. Representative images showing respective xenograft tumours at day 36 post subcutaneous injection ( $n=3$ per group). (b,c) Tumour sizes measured and depicted as tumour volume (b) or tumour weight (c). Error bars represent s.e.m. ( ${ }^{\star \star} P$ values $=0.005,{ }^{\star \star \star} P$ values $=0.0005$, respectively, Student's $t$-test). (d) Western blot analysis in mouse xenografts from a showing the expression of indicated proteins. (e) Agrin knockdown MHCC-LM3 cells were stably transfected with rat full-length Agrin-GFP or vector-GFP control. Western blot showing the expression of Agrin/Agrin-GFP, GFP expression and $\beta$-actin (as a loading control).

(f) Cells $\left(5 \times 10^{6}\right)$ derived from e were subcutaneously injected in nude mice as in a to establish tumours. Representative tumours and their volume are depicted graphically; $n=6$ (for each group). Error bar represents s.e.m. ( ${ }^{\star \star} P$ value $=0.002,{ }^{\star \star \star} P$ value $<0.0001$, respectively, Student's $t$-test).

(g) Western blot analysis for Agrin expression in control shRNA and Agrin shRNA-transduced Hep3B cells with $\beta$-actin as a loading control (left panel). Cells $\left(1 \times 10^{6}\right)$ were then intrahepatically inoculated in mice. Representative image of hepatic nodules 6 weeks post-hepatic injection (right panel), $n=5$ (per group). Black arrows indicate hepatic nodules. Scale bar, $10 \mathrm{~mm}$. (h) The hepatic tumour nodules ( $>1 \mathrm{~mm}$ in size) in control and Agrin-depleted Hep3B cell injected livers are quantified ( ${ }^{\star \star \star} P$ value $=0.0002$, Student's $t$-test).

to investigate whether the observed roles of Agrin in HCC is mediated through Lrp4-MuSK or occurred independently by alternative pathways. Reverse transcription PCR analysis with primers flanking $\mathrm{Z}$ exons revealed high expression of Agrin $\mathrm{Z}$ transcripts in multiple HCC cell lines (Supplementary Fig. 13a,b). Moreover, Lrp4 and MuSK are also widely expressed in a panel of HCC cell lines (Supplementary Fig. 14a). MuSK and Lrp4 knockdown in MHCC-LM3 cells decreased proliferation and induced morphological changes similar to those observed upon Agrin depletion (Supplementary Fig. 14b,c). As Agrin depletion strongly reduced tyrosine phosphorylation of MuSK (Fig. 6e), we investigated the role of Agrin-Lrp4-MuSK complex in HCC. Interestingly, the co-IP of MuSK and Lrp4 in control cells was compromised in Agrin-depleted cells (Fig. 6f), suggesting that interaction between MuSK and Lrp4 occurs in an Agrindependent manner as reported previously ${ }^{39}$. Although Agrin does not directly interact with $\mathrm{MuSK}^{39-41}$, its association with Lrp4 stimulated the presence of MuSK in the Agrin-Lrp4 complex, which was decreased by $83 \%$ upon Lrp4 depletion
(Fig. 6g), indicating that the formation of Agrin-Lrp4-MuSK complex is due to Lrp4-dependent MuSK activation by Agrin. We next deciphered whether Agrin-Lrp4-MuSK complex indirectly signals to FAs. Indeed, IP with MuSK antibody co-recovered FAK, indicating an indirect presence of FAK in the Agrin-Lrp4MuSK complex, which was reduced by $96 \%$ upon Agrin depletion but was partly restored by addition of soluble Agrin (Fig. 6h). In addition, MuSK depletion decreased the interaction of FAK with Agrin by $92 \%$ (Fig. 6i). MuSK and Lrp4 depletion also significantly reduced cellular invasion rates by $68 \%$ and $52 \%$ compared with control cells, respectively (Fig. 6j). Importantly, overexpression of Lrp4 and MuSK in HCC tumours (8 out of 11 HCC patients; Supplementary Fig. 14d) is consistent with the notion that Agrin functions through the Lrp4-MuSK complex to activate FAK in driving the oncogenic programme of HCC cells.

Agrin's role in in vivo tumorigenesis. Having mechanistically deciphered the oncogenic properties of Agrin in vitro, we next 
a
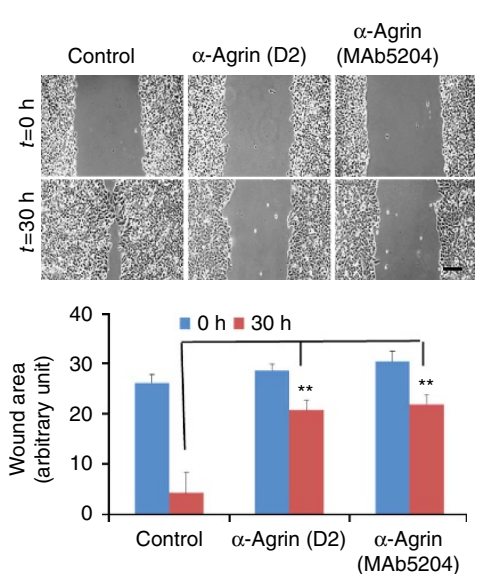

f

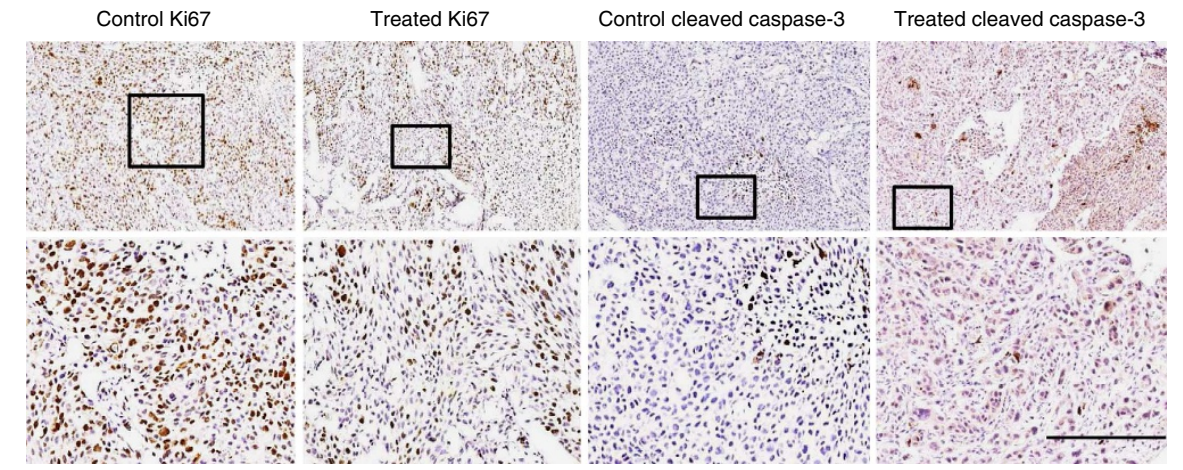

C

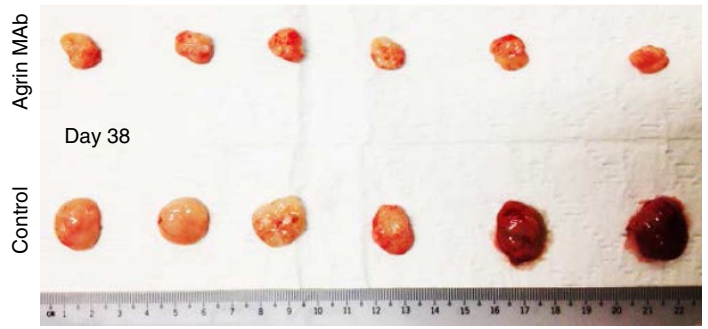

d

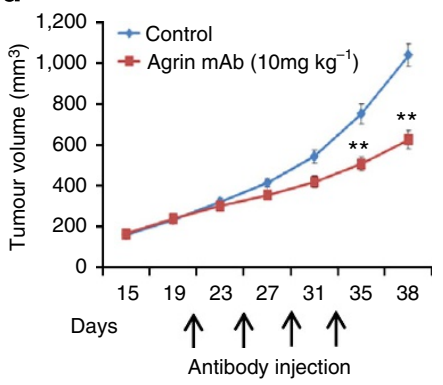

e

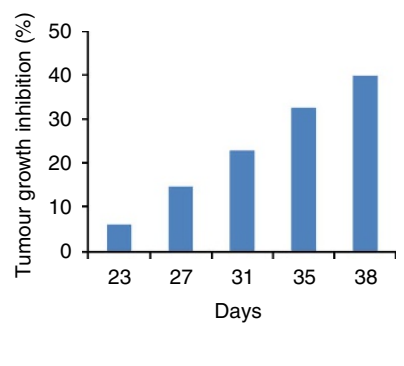

Figure 8 | Agrin functional blocking antibodies reduce tumorigenesis. (a) MHCC-LM3 cells were pre-treated with indicated Agrin antibodies $\left(10 \mu \mathrm{g} \mathrm{ml}^{-1}\right)$ for $12 \mathrm{~h}$ and then a wound-healing assay was performed in the presence of respective antibodies. Representative bright-field images are shown at indicated time points post scratching. Results are quantified using ImageJ software and error bars represent s.d. of mean area unhealed over time (in arbitrary unit) for three independent experiments performed in triplicates ( ${ }^{\star \star} P$ value $=0.004$, Student's $t$-test). Scale bar, $50 \mu \mathrm{m}$. (b) MHCC-LM3 cells were pre-treated with Agrin antibodies $\left(10 \mu \mathrm{g} \mathrm{ml}{ }^{-1}\right)$ for $12 \mathrm{~h}$ and lysates were subjected to western blot analysis for the indicated proteins. (c,d) Athymic nude mice were injected subcutaneously with MHCC-LM3 cells $\left(1 \times 10^{7}\right.$ cells per $\left.\mathrm{ml}\right)$ to develop tumours. After the tumours reached a size of $\sim 200 \mathrm{~mm}{ }^{3}$, MAb5204 antibody (dose $10 \mathrm{mg} \mathrm{kg}^{-1}$ ) against Agrin ( $n=6$ mice) or PBS control ( $n=6$ mice) was injected four times at an interval of 4 days. Tumour sizes shown after mice were killed at day 38. Mean tumour volume is represented in d. Error bars represent s.e.m. ( ${ }^{\star \star P} P$ values $<0.001$, Student's $t$-test). (e) Tumour growth inhibition depicted with respect to PBS control on the days subsequently after antibody injection. (f) Immunohistochemical analyses of antibody-treated or PBS-treated control tumours stained for proliferation marker (Ki67) and apoptosis marker (cleaved caspase-3). Representative bright-field images are shown ( $n=3$ mice per group). Boxed areas are represented as enlarged panels. At least six different control or antibody-treated tumour sections were analysed and quantified using LEICA image software for Ki67 and cleaved caspase-3 staining positivity. The percentage of positive staining cells are plotted and error bars represent s.d. of mean values of at least six different sections ( ${ }^{\star} P$ value $=0.02,{ }^{\star \star} P$ value $=0.003$, respectively, Student's $t$-test). Scale bar, $100 \mu \mathrm{m}$.

examined whether silencing Agrin affects tumour development in vivo. While mice injected subcutaneously with control MHCCLM3 cells developed solid tumours $>1,000 \mathrm{~mm}^{3}$ in volume and weight (at day 36), Agrin-depleted xenografts were significantly smaller in volume $\left(\sim 57 \mathrm{~mm}^{3}\right)$ and weight $($ Fig. $7 \mathrm{a}-\mathrm{c})$. Western blot analysis revealed reduced levels of Ki67, pY397FAK, pAkt and vimentin but increased levels of cleaved caspase-3 in Agrindepleted tumours (Fig. 7d). To validate that the in vivo impact is truly due to Agrin silencing, we restored Agrin in knockdown cells by stably expressing a full-length rat Agrin-GFP construct (Fig. 7e). Agrin-GFP rescued cells have significantly increased in vivo tumour growth (Fig. 7f). Furthermore, the in vivo role of Agrin was also investigated in an orthotopic HCC tumour model as tissue microenvironment has a crucial role in carcinogenesis.
Six weeks post intrahepatic inoculation, mice injected with Agrin-depleted Hep3B cells developed significantly lesser hepatic nodules than control cells (Fig. 7g,h). Cumulatively, these results confirm the oncogenic potential of Agrin in vivo.

Agrin antibodies reduce signaling and tumour growth. The necessity of Agrin in tumorigenesis may represent it as a suitable therapeutic target in HCC. Therefore, we characterized two Agrin targeting antibodies (D2 antibody and MAb5204 antibody, respectively). Their antigenic epitopes are mapped within the 20$\mathrm{kDa}$ C-terminal fragment of rat Agrin (Supplementary Fig. 15a) and recognize full-length protein and C-terminal fragment (Supplementary Fig. 15b). Agrin antibodies strongly inhibited 
a
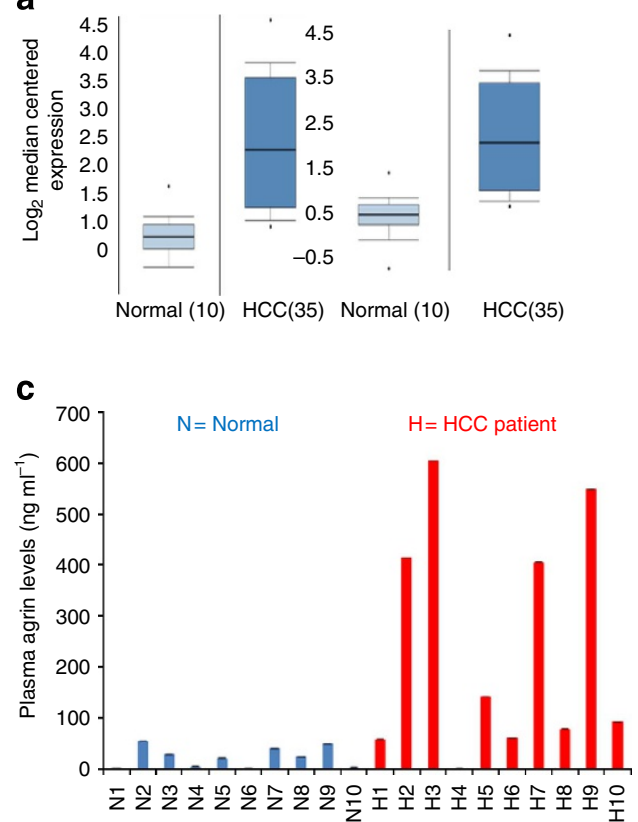

b

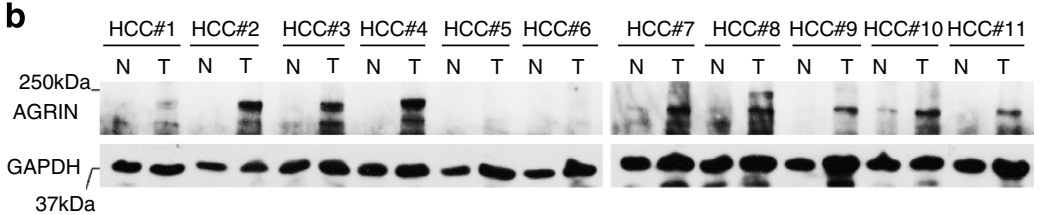

d

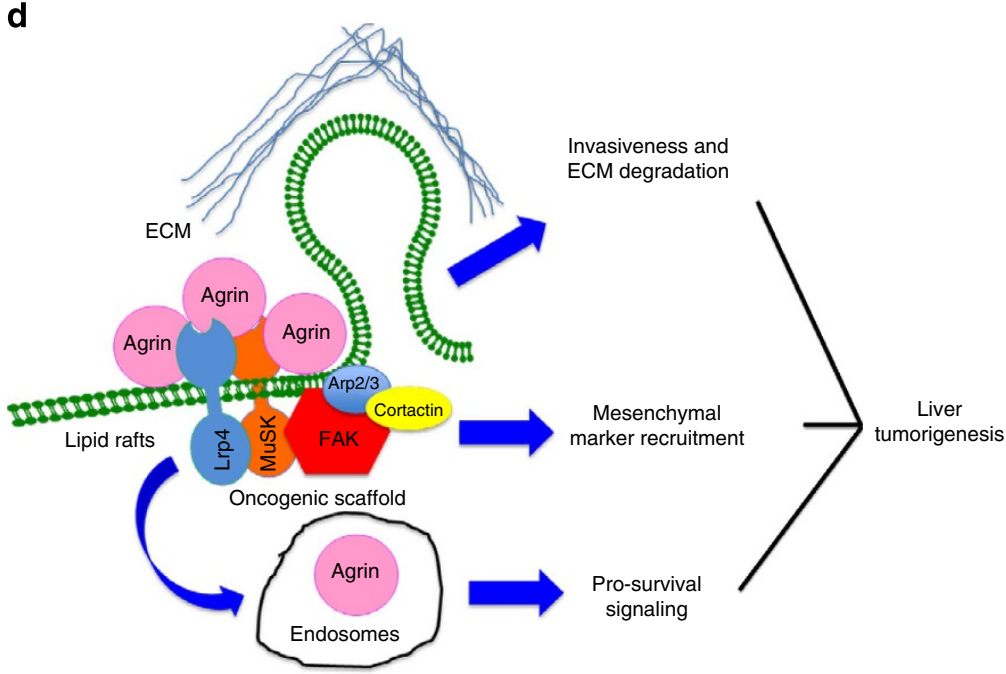

Figure 9 | Agrin is frequently overexpressed in HCC. (a) Gene expression analysis of Agrin using microarray (Oncomine) on normal liver versus HCC patient data sets. Pre-processed expression levels are $\log _{2}$ normalized and median centred ( $P$ values $=6.22 \mathrm{e}-6$ and $1.22 \mathrm{e}-7$, respectively). Error bars represent s.e.m. (b) Western blot analysis for Agrin in a cohort of liver cancer patients in Singapore. GAPDH was used as a loading control. (c) Detection of circulating Agrin in the plasma samples of healthy normal individuals and HCC patients by Agrin ELISA. Data represented as mean \pm s.d. (d) A working model describing the role of Agrin in HCC. Overexpression of secreted and cell surface Agrin triggers elevated binding to its receptors Lrp4 and promotes the formation of the Agrin-Lrp4-MuSK signalling complex, which activates FAs (FAK activation), Arp2/3 associated components and cortactin generating ruffling and invadopodia. This ECM sensor activity of Agrin is critical for sustaining FAK activity, cell motility, invasiveness, matrix degradation and subsequent mesenchymal marker recruitment to cell membrane. Internalized Agrin and its complex may also mediate signalling at the endosomal compartments. Cumulatively, these Agrin-mediated events are essential for hepatic tumorigenesis.

Agrin-induced MuSK phosphorylation in differentiated muscle C2C12 and MHCC-LM3 cells, suggesting their functional blocking activities (Supplementary Fig. 15c,d). As such, both antibodies significantly reduced cell migration by $\sim 77 \%$ as compared with isotype control (Fig. 8a). Agrin antibodies strongly suppressed pY397FAK, pAKT (Ser473), N-cadherin and vimentin expression while upregulating E-cadherin without affecting glypican-3 levels (Fig. 8b). Since MAb5204 showed greater in vitro effects, we examined its inhibitory role on tumour growth in a xenograft model. MAb5204 antibody treatment reduced the growth of pre-established tumours compared with PBS controls with $\sim 40 \%$ tumour growth inhibition (Fig. 8c-e). Consistently, treated tumours displayed reduced proliferation (Ki67) by $42 \%$ and increased apoptosis (cleaved caspase-3) by $87 \%$ (Fig. 8f), suggesting that MAb5204 antibody treatment reduces tumour growth.

Agrin is frequently overexpressed in HCC patients. To establish a clinical relevance of Agrin's role in HCC, we analysed HCC patient microarray data sets ${ }^{42,43}$. Consistent with our findings, we observed a 3.5- and 3.9-fold upregulation of Agrin (AGRN) mRNA in HCC patients (compared with normal liver tissues) in two independent data sets (Fig. 9a). Western blot analysis of matched normal (non-tumour; N) and HCC (liver-tumour; T) specimens revealed a significant upregulation of Agrin (9 out of 11 HCC patients) in cancer (Fig. 9b and Supplementary Table 3). Similarly compared with normal liver tissues, Agrin overexpression was also observed across various stages of HCC (Supplementary Fig. 16a,b and Supplementary Table 3).
Furthermore, circulatory plasma Agrin levels are higher in HCC patients when compared with normal individuals (Fig. 9c and Supplementary Table 3). These results confirm Agrin overexpression in tumour tissues and increased levels of circulating Agrin in HCC.

\section{Discussion}

Multiple lines of evidence presented here identify a unique ECM sensor activity of Agrin in providing oncogenic cues that promote liver tumorigenesis. We demonstrate Agrin's role in in vitro and in vivo liver tumorigenesis by stimulating cellular motility, FA integrity and EMT. We also validated the frequent upregulation of Agrin in clinical HCC specimens. Since cell surface proteins are the best known therapeutic targets in most cancers, our results suggest that targeting Agrin may be a potential approach to suppress HCC.

Proteomic screens offer unbiased approach to identify candidates in many cancer types ${ }^{44}$. Since quantitative membrane proteomics is rare in liver cancer, our SILAC analysis identifying differentially expressed cell surface proteins was designed accordingly. Based on our data, Agrin appears to be secreted and enriched in the plasma membrane (especially the lipid rafts) from where it can be actively endocytosed. As Agrin forms a complex with Lrp4-MuSK in HCC cells, it is likely that the endocytosis also occurs as the complex. Such endocytic events are often associated with signal amplification in many cancers ${ }^{45}$.

The EMT programme is activated when invading tumour cells breach through the underlying basement membrane and $\mathrm{ECM}^{46}$. However, how the basement membrane components regulate 
EMT is unknown. Interestingly, Agrin is reported as a basement membrane protein 7,28 . Several lines of evidence including changes in cell morphology, reversal of EMT characteristics and loss of invasiveness and clonogenecity upon Agrin knockdown are suggestive of Agrin's role in tumorigenesis. Conversely, Agrin rescue experiments by either expressing exogenous protein or addition of soluble Agrin restored migration, invasion and mesenchymal characteristics.

The molecular mechanism of Agrin's action was investigated. First, it was revealed that Agrin coupled to Arp2/3 regulates invadopodia formation required for ECM degradation and invasion. Second, Agrin activates the integrin-FAK pathway and maintains FA integrity. Agrin silencing or antibody treatment hampers FA integrity while Agrin re-expression restores FAs in knockdown cells. Since FAK controls mesenchymal characteristics bestowing adhesion and invasiveness in cancer cells ${ }^{34}$, Agrin-FAK axis probably drives EMT programme in HCC, corroborated by our FAK complementation evidence in Agrindeprived cells. As integrin-FAK signalling also directs proliferation for metastatic cancer cells ${ }^{49}$, it can be speculated that Agrin provides stimulatory signals to augment and sustain FAK activity and proliferation during tumour growth and extravasation. Instances where Agrin-depleted cells displayed poor FA activity, less invasiveness and synergistic inhibition of the Agrin-FAK pathway having robust growth inhibitory effects shed light to the above hypothesis. Elevated Agrin expression leads to enhanced binding and signalling through Lrp4 and MuSK. Anti-proliferative effects, loss of FA interactions and invasiveness upon Lrp4-MuSK depletion in the presence of Agrin strongly support that Agrin-Lrp4-MuSK complex may indirectly mediate signalling of Agrin to FAs and form a critical oncogenic axis (a working model presented in Fig. 9d). Moreover, reduced activity of Arp $2 / 3$ complex driving actin-induced membrane protrusions, FA alignment and migration observed upon Agrin depletion can be significantly reversed by Agrin supplementation in the ECM. Therefore, Agrin serves as the unique link via Lrp4MuSK between extracellular surface and FAK signalling critical for regulating tumorigenesis.

On the clinical perspective, elevated Agrin levels in HCC patient microarray data sets, tumour tissues and circulation of HCC patients cumulatively emphasize a strong role of Agrin in liver cancer. Agrin depletion hampered oncogenic signalling and tumour growth in xenograft and orthotopic models that is rescued by Agrin re-expression or addition of soluble Agrin, suggesting the tumour initiating and maintenance properties of Agrin. Low Agrin expression in normal livers and the observation that Agrin antibodies reduced pre-established tumour growth offer the possibility of Agrin as a potential therapeutic target by antibody therapy. Moreover, the increased plasma Agrin levels may also be exploited to develop diagnostic strategy for HCC in the future.

In conclusion, we reveal Agrin as an important factor activating and coordinating cellular adhesion, migration and invasiveness of HCC cancer cells. Although the role(s) of surface proteoglycans in promoting liver tumorigenesis are unclear, therapeutically targeting proteoglycans is gaining immense potential in $\mathrm{HCC}^{48}$. Therefore, targeting Agrin may augment additional HCC therapeutic strategies in the future. Moreover, our identification of Agrin's biological role sheds light on the broad cellular mechanisms as to how proteoglycans may regulate HCC.

\section{Methods}

Cell lines. The human HCC cell lines Hep3B, HepG2 and Huh-7 cells were purchased from American Type Cell Culture (Manassas, VA) and cultured in recommended media. The immortalized non-tumorigenic human hepatocyte cell line MIHA was kindly gifted by Dr J.M. Luk ${ }^{49}$. All other HCC cell lines were routinely maintained in Dr Kam-Man Hui laboratory (National Cancer Center, Singapore). Cells were cultured as per standard conditions described previously $y^{50}$ C2C12 mouse myoblasts were cultured in DMEM supplemented with $10 \%$ fetal bovine serum (FBS) and were differentiated in DMEM containing $2 \%$ horse serum for another 3 days ${ }^{51}$.

Patient data analysis. The collection of tumour and adjacent normal liver tissues and plasma from HCC patients were approved by the SingHealth Institutional Review Board (CIRB Ref:2007/447/B). All the tissues studied were provided by The SingHealth Tissue Repository. Written informed consent was obtained from all participating patients. Clinical and histopathological data provided to the researchers were rendered anonymous. Normal liver and HCC tissue microarray slides were obtained from US Biomax, Inc. (catalogue no. BC03116). The disease information of patients are provided in Supplementary Table 3.

Antibodies and reagents. Agrin (D2) and rabbit (H-300), Lrp4, Cdc42, N-WASP (western blot (WB) dilution-1:100) and anti-GFP (WB dilution-1:1,000) antibodies were obtained from Santa Cruz Biotechnology Inc., Santa Cruz, CA), Antibodies against $\mathrm{Na}+/ \mathrm{K}+$ ATPase, Rab5, integrin $\beta 1$, cleaved caspase- 3 , total FAK, cortactin, pSrc, total Src, pPI3-K, total PI3-K, pAkt, total Akt, pERK1/2 and total ERK1/2 were obtained from Cell Signaling Technology (dilution for WB1:1,000). Antibodies against pFAK pY397, caveolin-1, Ki67, E-cadherin, N-cadherin and Vimentin were purchased from BD Biosciences, San Jose, CA (WB dilution-1:500). PF-562271, glypican-3, flotillin-1, Arp2/3 subunit 1B, pFAK (pY397), Snail-1 and MuSK antibodies were obtained from Abcam (dilution for WB-1:500). Phospho-tyrosine monoclonal antibody 4G10, Agrin monoclonal antibody MAb5204, p34Arc, GFP and EGFP antibodies were obtained from Millipore, Billerica, MA (WB dilutions-1:1,000). Agrin polyclonal rabbit antibody, OKT-9 hybridoma cells producing anti-CD-71 antibody and fibronectin antibody were obtained from Sigma, St Louis, MO. Recombinant rat Agrin protein residues was obtained from R\&D systems and My Biosource, Inc, San Diego, CA. Recombinant CTxB, Alexa 488 and 555 conjugated secondary antibodies were from purchased Molecular Probes, Invitrogen, Carlsbad, CA.

In vivo tumorigenesis. Xenograft tumorigenesis: Six- to eight-week-old female nude mice were inoculated subcutaneously in the left and right hind flanks with $1 \times 10^{7}$ cells per $\mathrm{ml}$ suspended in Matrigel (1:1). Tumour development was monitored over a period of 30-36 days before the mice were killed for further analysis. Tumour volume $\left(\mathrm{mm}^{3}\right)$ is calculated by the formula $V=L \times B^{2} / 2$, where $V=$ volume of tumour, $L=$ length of tumour and $B=$ breadth of tumour measured in $\mathrm{mm}$ scale. Intrahepatic tumorigenesis was performed as follows ${ }^{52}$ : a midline abdominal incision was made to expose the lateral liver lobes. Approximately, $1 \times 10^{6}$ cells were directly delivered into liver parenchyma. Six weeks later, mice were killed and hepatic nodules $>1 \mathrm{~mm}$ in diameter were counted. All animal care and handling were in compliance with Institutional Animal Care and Use Committee at Institute of Molecular and Cell Biology, A*STAR, Singapore.

Generation of knockdown and rescue cells. A lentiviral pool encoding shAgrin (catalogue no. sc-29652-V) and scramble shRNA control (catalogue no. sc-108080) were purchased from Santa Cruz Biotechnology Inc. Viral transduction was performed as per manufacturer's protocol. Agrin shRNAs comprises a pool of three different shRNA hairpins specifically targeting human Agrin. Their sense sequences are $5^{\prime}$-CAGGAGAAUGUCUUCAAGATT- $3^{\prime}, 5^{\prime}$-CGACGUGUGCUGU GAAGAATT- $3^{\prime}$ and $5^{\prime}$-CGACCUCUUCCGGAAUUCATT- $3^{\prime}$, respectively. Briefly, $30 \mu$ l of viral particles was used to infect MHCC-LM3 cells overnight along with $0.5 \mu \mathrm{g} \mathrm{ml}^{-1}$ polybrene in complete media. Next day, medium was changed and cells were re-infected for $2 \mathrm{~h}$ and monitored for 2 more days before selection in media containing puromycin $\left(2 \mu \mathrm{g} \mathrm{ml}^{-1}\right)$ for at least two doubling times. After 3 days, knockdown was verified by a western blot analysis using Agrin-specific antibody. Agrin rescue in previously knocked down MHCC-LM3 cells were performed by nucleofection of a full-length rat Agrin-GFP construct that is not targeted by human shRNA sequences as per standard protocol. Forty-eight hours post nucleofection, GFP cells were selected using $400 \mathrm{\mu g} \mathrm{ml}^{-1}$ neomycin (G418) for 10 days. A western blot confirming stable Agrin-GFP expression was performed.

siRNAs plasmids and transfections. For siRNA-mediated knockdown, ONTARGET plus SMARTpool of siRNA against human Agrin (no. L-031716-000050), human Lrp4 (no. L-027194-02-0020), human PTK2 (no. L003164-0020) and scramble control (no. D-001210-01-20) were obtained from Dharmacon (Thermo Fisher Scientific). siRNA targeting MuSK (no. AM16704) and ArpC2/p34 (no. AM16708) were obtained from Ambion Life Technologies. Cells were transfected using RNA-iMAX Lipofectamine 2000 (Invitrogen). A western blot analysis verifying target protein knockdown was performed after $72 \mathrm{~h}$ post transfection. Full-length rat Agrin-GFP, C-terminal and N-terminal Agrin-GFP were kindly gifted by Dr Matthew P. Daniels, National Institutes of Health, Bethesda, MD, USA $^{53}$. EGFP-FAK and EGFP control vectors were obtained from Addgene. 
Biotinylation and cell surface protein extraction. Cell surface proteins were biotinylated and extracted using the Pierce Cell Surface Protein Isolation kit (Pierce, IL) as per manufacturer's protocol. Briefly, cells labelled with $0.25 \mathrm{mg} \mathrm{ml}^{-1}$ cleavable biotinylation reagent (Sulfo-NHS-SS-Biotin) for $30 \mathrm{~min}$ at $4{ }^{\circ} \mathrm{C}$ and quenched by adding $2 \mathrm{ml}$ of quenching solution. Post washing, cells were harvested in lysis buffer (Pierce), sonicated in ice for $30 \mathrm{~min}$ followed by centrifugation at $10,000 \mathrm{~g}$ for $2 \mathrm{~min}$. Biotin-labelled proteins were affinity purified with streptavidin Agarose and eluted with SDS-polyacrylamide gel electrophoresis (SDS-PAGE) sample buffer $(62.5 \mathrm{mM}$ Tris- $\mathrm{HCl}, \mathrm{pH} 6.8,1 \%$ SDS, $10 \%$ glycerol and $50 \mathrm{mM}$ dithiothreitol). Flow-through fractions were also analysed.

SILAC-based mass spectrometry analysis. Hep3B and MIHA cells were cultured in DMEM for SILAC (Thermo Fisher Scientific) supplemented with 10\% dialysed FBS either containing normal isotopes of L-lysine- $\left({ }^{12} \mathrm{C}_{6}^{14} \mathrm{~N}_{2}\right)\left(\mathrm{K}^{0}\right)$ and L-arginine$\left.{ }^{(12} \mathrm{C}_{6}^{14} \mathrm{~N}_{4}\right)\left(\mathrm{R}^{0}\right)\left(\mathrm{K}^{0} \mathrm{R}^{0}\right.$ - light') or stable isotope L-lysine- $\left({ }^{13} \mathrm{C}_{6}^{15} \mathrm{~N}_{2}\right)\left(\mathrm{K}^{8}\right)$ and L-arginine- $\left({ }^{13} \mathrm{C}_{6}^{15} \mathrm{~N}_{4}\right)\left(\mathrm{R}^{10}\right)\left(\mathrm{R}^{10} \mathrm{~K}^{8}\right.$-'heavy') for at least six doublings to ensure efficient incorporation of labelled amino acids. Cells were subsequently biotinylated and cell surface proteins were extracted as described above, and equal amount of cell lysates from light $\left(\mathrm{K}^{0} \mathrm{R}^{0}\right)$ and heavy $\left(\mathrm{R}^{10} \mathrm{~K}^{8}\right)$ were mixed and separated on a $10 \%$ SDS-PAGE. Extracted peptides were subjected to LC-Orbitrap MS analysis (Fig. 1a). Equal amounts of protein lysates from heavy- and light biotin-labelled cell lysates were mixed, and $80 \mu \mathrm{g}$ proteins were separated on a one-dimensional gradient (4-12\%) Nu-Page Novex Bis-Tris gel (Invitrogen) and digested with trypsin described previously ${ }^{54}$. The samples were analysed on an Orbitrap XL (Thermo Fisher Scientific) full-scan mass spectra. Identification and quantification of peptides were performed using mascot version 2.2 (Matrix Science, London, U.K.) as described previously ${ }^{54}$.

Western blot analysis. Cells were washed once with ice-cold PBS, lysed in cold lysis buffer ( $150 \mathrm{mM} \mathrm{NaCl}, 50 \mathrm{mM}$ Tris- $\mathrm{HCl}$ pH 7.3, $0.25 \mathrm{mM}$ EDTA pH 8.0, 1\% sodium deoxycholate, $1 \%$ Triton X-100, $0.2 \%$ sodium fluoride and $0.1 \%$ sodium orthovanadate supplemented with protease inhibitor cocktail (Roche Applied Biosciences). Cell lysate was centrifuged at 13,000 r.p.m. for $30 \mathrm{~min}$, boiled in $2 \times$ sample buffer, separated on either $7.5,10$ or $4-20 \%$ gradient SDS-PAGE and blotted onto nitrocellulose membrane. The membrane was blocked in 5\% skimmed milk in PBS containing $0.1 \%$ Tween-20, probed with primary antibody followed by appropriate secondary antibody conjugated with horseradish peroxidase (Pierce) and immunoreactive bands were visualized by enhanced chemiluminescence super signal pico (Pierce). Bands were quantified using ImageJ software. For phosphoproteins, bands were normalized to their respective total protein levels, while for other panels bands were normalized to a loading control ( $\beta$-actin). The respective values in arbitrary units are either shown in the panels as 'relative levels' or plotted graphically as fold change. The uncropped scanned full gels are shown in Supplementary Fig. 17.

Immunoprecipitation. Cells were lysed in lysis buffer (20 mM Tris-HCl, $\mathrm{pH} 7.5$, $150 \mathrm{mM} \mathrm{NaCl}, 1 \%$ Triton X-100 and $1 \mathrm{mM}$ PMSF with complete EDTA-free protease inhibitor mixture (Roche)). The lysate was incubated on ice for $30 \mathrm{~min}$ and cleared by centrifugation at 13,000 r.p.m. for $30 \mathrm{~min}$ at $4{ }^{\circ} \mathrm{C}$. IP was performed at $4{ }^{\circ} \mathrm{C}$ with $5 \mu \mathrm{g}$ of antibody in the presence of either protein $\mathrm{A}$ or protein G-Sepharose 4 Fast Flow (GE Healthcare) for $4 \mathrm{~h}$ at $4{ }^{\circ} \mathrm{C}$ in a rocker. The Sepharose bound proteins was then washed three times with cell lysis buffer and three times with cold PBS. Bound proteins were eluted with $2 \times$ Laemmli sample buffer, resolved by SDS-PAGE for subsequent Western blotting. Quantification of IP protein band was normalized to that of bait protein and represented as percentage pulldown (\% IP).

Reverse transcription PCR analysis. Total RNA was isolated from indicated cell lines using Qiagen RNeasy minikit as per the manufacturer's protocol. An amount of $10 \mu \mathrm{g}$ RNA aliquots were then reverse transcribed using High Capacity cDNA reverse transcription kit, Applied Biosystems Inc., as per standard protocols. cDNA was used as template for PCR, and PCR products were separated on a $1.5 \%$ agarose gel. Agrin $\mathrm{Z}$ exon flanking primers: forward $5^{\prime}$-ACACCGTCCTCAACCTGAAG-3' and reverse $5^{\prime}$-CCAGGTTGTAGCTCAGTTGC- $3^{\prime}$. Expected product size $=453$ bp. Vimentin primers: forward $5^{\prime}$-TCCAGCAGCTTCCTGTAGGT- $3^{\prime}$ reverse $5^{\prime}$-CCCTCACCTGTGAAGTGGAT- $3^{\prime}$, Arp2 forward 5'-GTAATGTTTGAAAC TTACCAG-3', reverse $5^{\prime}$-CGATACCAAGGAATACCGAC-3'; Arp3 forward $5^{\prime}$-AGAGATTTGAAAAGAACTGTAG- $3^{\prime}$ reverse 5-'ACTGGTCCAACACTCTT GTC-3', GAPDH: forward 5'-GAGCCACATCGCTCAGAC-3' reverse $5^{\prime}$-CTTC TCATGGTTCACACCC- $3^{\prime}$, respectively.

Agrin ELISA. Secreted Agrin were determined in normal and HCC patient plasma using an Agrin ELISA kit (MyBiosource, Inc.). Briefly, $50 \mu \mathrm{l}$ of provided Agrin standard or diluted plasma were added to 96-well plates coated with Agrin antibodies. A volume of $100 \mu \mathrm{l}$ of secondary antibody conjugated to horseradish peroxide were then added to the wells and incubated for $1 \mathrm{~h}$ at $37^{\circ} \mathrm{C}$. Post washing, $100 \mu \mathrm{l}$ of substrate was added to each well followed by $50 \mu \mathrm{l}$ stop solution.

Absorbance was measured at $450 \mathrm{~nm}$ wavelength. Agrin levels were calculated from a standard curve. Each patient samples were analysed in quadruplex. Data represented as mean \pm s.d.

Cytosolic soluble and membrane fractionation. Cells were harvested in homogenization buffer $(20 \mathrm{mM}$ Tris-HCl, $\mathrm{pH} 7.5,150 \mathrm{mM} \mathrm{NaCl}$ and $1 \mathrm{mM}$ PMSF with complete EDTA-free protease inhibitor mixture (Roche)). The cell suspension homogenized through a $29-\mathrm{G}$ needle 10 times on ice was briefly centrifuged at 2,000 r.p.m. for $5 \mathrm{~min}$ at $4^{\circ} \mathrm{C}$. The clarified lysate was then centrifuged at 80,000 r.p.m. using the TLA120.1 rotor (Beckman Coulter) for $1 \mathrm{~h}$ at $4{ }^{\circ} \mathrm{C}$. Cytosol was collected and the membrane pellet was washed once with cytosol buffer and then centrifuged at 80,000 r.p.m. for $30 \mathrm{~min}$ at $4^{\circ} \mathrm{C}$ once more. The membrane pellet was dissolved in lysis buffer and centrifuged at 80,000 r.p.m. for $1 \mathrm{~h}$ at $4{ }^{\circ} \mathrm{C}$ for the third time. The solubilized membrane proteins were then collected.

Lipid raft/caveolae membrane isolation. Lipid raft/caveolae extraction was performed as per the instructions of lipid raft/caveolae isolation kit (Sigma) and previously published protocols ${ }^{55}$. Briefly, confluent cell lines were lysed in $0.5 \mathrm{M}$ sodium bicarbonate lysis buffer ( $\mathrm{pH} 11.0$ ) containing protease inhibitor cocktail (Roche) for $15 \mathrm{~min}$ on rocker. Cell lysate were homogenized in a Dounce homogenizer (10 strokes) and sonicated for $10 \mathrm{~s}$ at $4{ }^{\circ} \mathrm{C}$. A discontinuous gradient (0-35\%) of optiprep and lysis buffer was made, and $2 \mathrm{ml}$ of cell lysate and optiprep (35\%) was placed at the bottom of a centrifuge tube with each layer pre-ceding on top. The tubes were centrifuged at 40,000 r.p.m. for $4 \mathrm{~h}$ using a Beckmann SWI-55 rotor. A volume of $1 \mathrm{ml}$ fractions were collected from the top and analysed by Dot and western blots.

Agrin internalization assay. Hep3B cells were seeded in eight-well chamber slides (Nalge Nunc, Inc.). Confluent cells $(70-80 \%)$ were treated with $3 \mu \mathrm{g} \mathrm{ml}^{-1}$ Agrin antibody (Santa Cruz Biotechnology, Inc.) and $0.5 \mu \mathrm{g} \mathrm{ml}^{-1}$ CTxB subunit conjugated with Alexa 488 (green) in chilled complete medium for $1 \mathrm{~h}$ at $4{ }^{\circ} \mathrm{C}$ on ice. Subsequently, the antibody was washed away and cells were replenished with complete media for indicated time points at $37^{\circ} \mathrm{C}$. Cells were fixed after indicated time points with $4 \%$ paraformaldehyde for $15 \mathrm{~min}$ at room temperature (RT) and processed for immunofluorescence analysis using appropriate secondary Alexa Fluor-labelled antibodies (Molecular Probes)

Immunofluorescence confocal microscopy. Cells grown either on coverslips (Thermo Fisher Scientific) or in eight-well chamber slides (Nalge Nunc, Inc.) were washed two times with PBS supplemented with $1 \mathrm{mM} \mathrm{CaCl} 2$ and $1 \mathrm{mM} \mathrm{MgCl}$ (PBSCM). They were fixed with 4\% paraformaldehyde in PBSCM for $15 \mathrm{~min}$ at RT washed five times at 5-min intervals using PBSCM and permeabilized with $0.1 \%$ Triton X-100 in PBSCM for 5 min at RT. The cells were then immunostained with appropriate primary antibodies diluted in fluorescence dilution buffer (PBSCM with $5 \% \mathrm{FBS}$ and $2 \%$ bovine serum albumin) for $1 \mathrm{~h}$ at RT and washed five times with $0.1 \%$ Triton X-100 PBSCM at 5-min intervals. Secondary antibodies conjugated to either Alexa 488 (green) or Alexa 595 (red) were diluted appropriately in fluorescence dilution buffer and incubated at RT for $1 \mathrm{~h}$. The coverslips were washed again with $0.1 \%$ Triton X-100 PBSCM five times for 5-min each and were mounted on microscopic slides with Vectashield mounting medium containing 4,6-diamidino-2-phenylindole (DAPI; Vector Laboratories). Confocal microscopy was performed with either an Axioplan II microscope (Carl Zeiss, Inc.) equipped with Zeiss confocal scanning optics or Olympus Fluoview 1000 confocal microscope (Olympus).

Immunohistochemistry. Paraffin-embedded tissue sections were deparaffinized in Bond Dewax solution, rehydrated through $100 \%$ ethanol to $1 \times$ Bond Wash solution, antigenic epitope retrieval performed at $\mathrm{pH} 6.0$ and blocked in $10 \%$ goat serum for $30 \mathrm{~min}$. Slides were then incubated with primary antibodies in Bond diluent solution for $45 \mathrm{~min}$, washed three times, polymerized for $10 \mathrm{~min}$ and washed again for at least four times. Nuclei were counterstained with haematoxylin for $5 \mathrm{~min}$, rinsed in deionized water and mounted in mounting medium. Slides were visualized under a bright-field microscope using Leica imaging software.

Live-cell imaging. Control or Agrin shRNA-tranduced MHCC-LM3 cells were cultured in glass bottom Petri dishes (MatTek Corporation) for 12-16h before imaging live at $37^{\circ} \mathrm{C}$ using the differential interference contrast filter in a confocal microscope (Olympus). For paxillin-GFP imaging, cells were trypsinized and plated as in adhesion assay and imaged $3 \mathrm{~h}$ post plating. Each image shown in panels corresponds to a 1-min interval. Data were analysed using the Fluoview software.

Wound-healing assay. Cell migration was assessed by wound-healing assays. Briefly, confluent MHCC-LM3 cells either transduced with control shRNA or Agrin shRNA plated on six-well plates were wounded by manual scratching with a 200- $\mu$ l pipette tip. Subsequently, cells were washed with PBS and incubated at $37^{\circ} \mathrm{C}$ in complete media. At the indicated time points, phase contrast images at specific wound sites were taken. 
Anchorage-independent growth in soft agar. A volume of $1.5 \mathrm{ml}$ of $0.5 \%$ agar (electrograde ultra-pure; Invitrogen, Carlsbad, CA) supplemented with DMEM, $10 \%$ FBS, was plated in six-well culture dishes as bottom agar. Three thousand cells were mixed with $1.5 \mathrm{ml}$ of $0.35 \%$ agar supplemented with DMEM, $10 \% \mathrm{FBS}$, and plated on top of the bottom agar. A volume of $1 \mathrm{ml}$ of media was added on top of solidified agar layers and colonies were allowed to grow in incubator at $37^{\circ} \mathrm{C}$ for 10-12 days. Images of cell colonies were observed in an inverted microscope.

Three-dimensional sphere culture. A volume of $45 \mu \mathrm{l}$ of growth factor reduced Matrigel (BD Biosciences) was added to eight-well chamber slides and solidified in $37^{\circ} \mathrm{C}$ incubator for $15 \mathrm{~min}$. MHCC-LM3 cells were trypsinized and resuspended in $400 \mu \mathrm{l}$ culture media containing $2 \%$ Matrigel to have 5,000 cells per well. The cells were grown in a $5 \% \mathrm{CO}_{2}$ humidified incubator for 10-12 days and nourished with medium every 3-4 days. Microscopic observation was performed to screen spheres.

Transwell migration and invasion assays. Cell invasion was determined using the 24-well chambers with 8 - $\mu \mathrm{m}$ pore polycarbonate membranes either uncoated (for migration) or pre-coated with Matrigel Basement Membrane Matrix (for invasion; BD Biosciences). The chambers were rehydrated in serum-free medium. Complete medium with $10 \%$ FBS $(700 \mu \mathrm{l})$ served as chemoattractant in the bottom chamber and $1 \times 10^{4}$ cells per ml cells were incubated for $24 \mathrm{~h}$ at $37^{\circ} \mathrm{C}, 5 \% \mathrm{CO}_{2}$. At the end of incubation period, cells invading membrane or Matrigel were washed and stained with $0.05 \%$ crystal violet solution and imaged in a bright-field microscope.

Fibronectin adhesion assay. Briefly, culture plates were coated with $10 \mu \mathrm{g} \mathrm{ml}^{-1}$ fibronectin for $1 \mathrm{~h}$ at $37^{\circ} \mathrm{C}$. Control or Agrin knockdown cells $\left(2 \times 10^{3}\right.$ cells ml $\left.{ }^{-1}\right)$ were trypsinized and plated on coated dishes. Adhered cells were analysed either by live-cell imaging for morphological changes at indicated time points or lysates were collected for western blot analysis.

Actin polymerization assay. Actin polymerization assay was performed according to actin polymerization biochem kit (catalogue no. BK003), Cytoskeleton, Inc. based on enhanced pyrene actin fluorescence principle. Briefly, $200 \mu \mathrm{l}$ of $0.4 \mathrm{mg} \mathrm{ml}^{-1}$ globular actin (G-actin) in actin buffer with ATP was added to Corning 96-well plate reader, shaken for $5 \mathrm{~s}$ and baseline fluorescence was read at $435 \mathrm{~nm}$ for $3 \mathrm{~min}$ for every min interval. A volume of $20 \mu \mathrm{l}$ of cell lysate $(20 \mu \mathrm{g}$ protein) and $20 \mu \mathrm{l}$ of actin polymerization buffer $(10 \times$ stock $)$ were added and plate was read constantly for $1 \mathrm{~h}$ with an interval of $1 \mathrm{~min}$. Means of triplicate data was expressed as arbitrary fluorescence units.

Gelatin degradation invadopodia assay. Invadopodia formation was assayed as per manufacturer's protocol using invadopodia assay kit (catalogue. no. ECM671), Millipore. Briefly, coverslides were coated with $0.2 \% \mathrm{w} / \mathrm{v}$ poly-L-lysine for $20 \mathrm{~min}$ at RT, washed three times with sterile PBS, followed by 15 min fixation with $0.5 \%$ glutaraldehyde at RT. After washing, slides were layered with Cy3-labelled gelatin and unlabelled gelatin in a ratio of 1:5 for $30 \mathrm{~min}$ at RT. Labelled gelatin matrix was sterilized with $70 \%$ ethanol for $30 \mathrm{~min}$. MHCC-LM3 $\left(2 \times 10^{3}\right)$ cells were layered onto labelled gelatin matrix and cultured for $12-16 \mathrm{~h}$ under standard tissue culture conditions. Cells were then fixed with 3.7\% paraformaldehyde (PFA) for $15 \mathrm{~min}$ at $\mathrm{RT}$ and processed for immunofluorescence. Cells with degraded matrix were imaged in a confocal microscope and quantified using ImageJ software. Microarray data mining and gene expression analysis. We analysed microarray
data sets containing normal and HCC patient samples (Oncomine) ${ }^{42,43}$. Since all of them were assayed on Affymetrics human-UG133 platform, the mean expression of Agrin using AGRN 212285_s_at as query was determined. Microarray data are pre-processed by Oncomine in a standardized way by $\log _{2}$ transformation and scaling the median value per microarray to 0 .

Statistical analysis. All experiments were performed in triplicates and repeated three times. For in vitro experiments, bar charts and graph represent mean values, and error bar indicates s.d. For in vivo experiments, error bars represent s.e.m. A paired 'two-tailed' Student's $t$-test was performed using GraphPad Prism software, and data were considered significant when ${ }^{*} P$ value $<0.05$, ${ }^{*} P$ value $<0.005$ and ${ }^{* * *} P$ value $<0.0005$, respectively.

\section{References}

1. Parkin, D. M., Bray, F., Ferlay, J. \& Pisani, P. Global cancer statistics, 2002. CA Cancer J. Clin. 55, 74-108 (2005).

2. Whittaker, S., Marais, R. \& Zhu, A. X. The role of signaling pathways in the development and treatment of hepatocellular carcinoma. Oncogene 29, 4989-5005 (2010).

3. Huynh, H. et al. Targeting receptor tyrosine kinase pathways in hepatocellular carcinoma. Anticancer Agents Med. Chem. 11, 560-575 (2011).
4. Kischel, P. et al. Cell membrane proteomic analysis identifies proteins differentially expressed in osteotropic human breast cancer cells. Neoplasia 10, 1014-1020 (2008).

5. Boersema, P. J., Geiger, T., Wisniewski, J. R. \& Mann, M. Quantification of the $\mathrm{N}$-glycosylated secretome by super-SILAC during breast cancer progression and in human blood samples. Mol. Cell. Proteomics. 12, 158-171 (2013).

6. Harsha, H. C., Molina, H. \& Pandey, A. Quantitative proteomics using stable isotope labeling with amino acids in cell culture. Nat. Protoc. 3, 505-516 (2008).

7. Tatrai, P. et al. Agrin, a novel basement membrane component in human and rat liver, accumulates in cirrhosis and hepatocellular carcinoma. Lab. Invest. 86, 1149-1160 (2006).

8. Somoracz, A. et al. Agrin immunohistochemistry facilitates the determination of primary versus metastatic origin of liver carcinomas. Hum. Pathol. 41, 1310-1319 (2010).

9. Tatrai, P. et al. Agrin and CD34 immunohistochemistry for the discrimination of benign versus malignant hepatocellular lesions. Am. J. Surg. Pathol. 33, 874-885 (2009).

10. Burgess, R. W., Skarnes, W. C. \& Sanes, J. R. Agrin isoforms with distinct amino termini: differential expression, localization, and function. J. Cell Biol. 151, 41-52 (2000).

11. Neumann, F. R. et al. An alternative amino-terminus expressed in the central nervous system converts agrin to a type II transmembrane protein. Mol. Cell. Neurosci. 17, 208-225 (2001).

12. McMahan, U. J. The agrin hypothesis. Cold Spring Harb. Symp. Quant. Biol. 55, 407-418 (1990).

13. Ferns, M., Deiner, M. \& Hall, Z. Agrin-induced acetylcholine receptor clustering in mammalian muscle requires tyrosine phosphorylation. J. Cell Biol. 132, 937-944 (1996).

14. Denzer, A. J., Hauser, D. M., Gesemann, M. \& Ruegg, M. A. Synaptic differentiation: the role of agrin in the formation and maintenance of the neuromuscular junction. Cell Tissue Res. 290, 357-365 (1997).

15. Kim, N. et al. Lrp4 is a receptor for Agrin and forms a complex with MuSK. Cell 135, 334-342 (2008).

16. Zhang, B. et al. LRP4 serves as a coreceptor of agrin. Neuron 60, 285-297 (2008).

17. Lin, L. et al. Induction of filopodia-like protrusions by transmembrane agrin: role of agrin glycosaminoglycan chains and Rho-family GTPases. Exp. Cell Res 316, 2260-2277 (2010).

18. Uhm, C. S., Neuhuber, B., Lowe, B., Crocker, V. \& Daniels, M. P. Synapse-forming axons and recombinant agrin induce microprocess formation on myotubes. J. Neurosci. 21, 9678-9689 (2001).

19. Denzer, A. J., Brandenberger, R., Gesemann, M., Chiquet, M. \& Ruegg, M. A. Agrin binds to the nerve-muscle basal lamina via laminin. J. Cell Biol. 137, 671-683 (1997).

20. Quail, D. F. \& Joyce, J. A. Microenvironmental regulation of tumor progression and metastasis. Nat. Med. 19, 1423-1437 (2013).

21. Frisch, S. M., Vuori, K., Ruoslahti, E. \& Chan-Hui, P. Y. Control of adhesiondependent cell survival by focal adhesion kinase. J. Cell Biol. 134, 793-799 (1996).

22. Cheng, N., Li, Y. \& Han, Z. G. Argonaute2 promotes tumor metastasis by way of up-regulating focal adhesion kinase expression in hepatocellular carcinoma. Hepatology 57, 1906-1918 (2013).

23. Shintani, Y., Hollingsworth, M. A., Wheelock, M. J. \& Johnson, K. R. Collagen I promotes metastasis in pancreatic cancer by activating c-Jun $\mathrm{NH}(2)$-terminal kinase 1 and up-regulating N-cadherin expression. Cancer Res. 66, 11745-11753 (2006).

24. Bagi, C. M. et al. Sunitinib and PF-562,271 (FAK/Pyk2 inhibitor) effectively block growth and recovery of human hepatocellular carcinoma in a rat xenograft model. Cancer Biol. Ther. 8, 856-865 (2009).

25. Stokes, J. B. et al. Inhibition of focal adhesion kinase by PF-562,271 inhibits the growth and metastasis of pancreatic cancer concomitant with altering the tumor microenvironment. Mol. Cancer Ther. 10, 2135-2145 (2011).

26. Tang, H. et al. Loss of Scar/WAVE complex promotes N-WASP- and FAK-dependent invasion. Curr. Biol. 23, 107-117 (2013).

27. Tancioni, I. et al. FAK Inhibition disrupts a beta5 integrin signaling axis controlling anchorage-independent ovarian carcinoma growth. Mol. Cancer Ther. 13, 2050-2061 (2014).

28. Bezakova, G. \& Ruegg, M. A. New insights into the roles of agrin. Nat. Rev. Mol. Cell. Biol. 4, 295-308 (2003).

29. Eckert, M. A. et al. Twist1-induced invadopodia formation promotes tumor metastasis. Cancer Cell 19, 372-386 (2011).

30. Tarone, G., Cirillo, D., Giancotti, F. G., Comoglio, P. M. \& Marchisio, P. C. Rous sarcoma virus-transformed fibroblasts adhere primarily at discrete protrusions of the ventral membrane called podosomes. Exp. Cell Res. 159, 141-157 (1985).

31. Linder, S. The matrix corroded: podosomes and invadopodia in extracellular matrix degradation. Trends Cell. Biol. 17, 107-117 (2007). 
32. Buccione, R., Orth, J. D. \& McNiven, M. A. Foot and mouth: podosomes, invadopodia and circular dorsal ruffles. Nat. Rev. Mol. Cell. Biol. 5, 647-657 (2004).

33. Murphy, D. A. \& Courtneidge, S. A. The 'ins' and 'outs' of podosomes and invadopodia: characteristics, formation and function. Nat. Rev. Mol. Cell. Biol. 12, 413-426 (2011).

34. Bhattacharya, R. et al. Recruitment of vimentin to the cell surface by beta3 integrin and plectin mediates adhesion strength. J. Cell. Sci. 122, 1390-1400 (2009).

35. Kuo, J. C., Han, X., Hsiao, C. T., Yates, 3rd J. R. \& Waterman, C. M. Analysis of the myosin-II-responsive focal adhesion proteome reveals a role for beta-Pix in negative regulation of focal adhesion maturation. Nat. Cell. Biol. 13, 383-393 (2011).

36. Wu, C. et al. Arp2/3 is critical for lamellipodia and response to extracellular matrix cues but is dispensable for chemotaxis. Cell 148, 973-987 (2012).

37. Alexander, N. R. et al. Extracellular matrix rigidity promotes invadopodia activity. Curr. Biol. 18, 1295-1299 (2008).

38. Pignatelli, J., Tumbarello, D. A., Schmidt, R. P. \& Turner, C. E. Hic-5 promotes invadopodia formation and invasion during TGF-beta-induced epithelial-mesenchymal transition. J. Cell Biol. 197, 421-437 (2012).

39. Zhang, W., Coldefy, A. S., Hubbard, S. R. \& Burden, S. J. Agrin binds to the $\mathrm{N}$-terminal region of Lrp4 protein and stimulates association between Lrp4 and the first immunoglobulin-like domain in muscle-specific kinase (MuSK). J. Biol. Chem. 286, 40624-40630 (2011).

40. Burden, S. J. SnapShot: neuromuscular junction. Cell 144, 826-826 e1 (2011).

41. Burden, S. J., Yumoto, N. \& Zhang, W. The role of MuSK in synapse formation and neuromuscular disease. Cold Spring Harb. Perspect. Biol. 5, a009167 (2013).

42. Roessler, S. et al. A unique metastasis gene signature enables prediction of tumor relapse in early-stage hepatocellular carcinoma patients. Cancer Res. 70, 10202-10212 (2010).

43. Wurmbach, E. et al. Genome-wide molecular profiles of HCV-induced dysplasia and hepatocellular carcinoma. Hepatology 45, 938-947 (2007).

44. Eustace, B. K. et al. Functional proteomic screens reveal an essential extracellular role for hsp90 alpha in cancer cell invasiveness. Nat. Cell. Biol. 6, 507-514 (2004).

45. Casaletto, J. B. \& McClatchey, A. I. Spatial regulation of receptor tyrosine kinases in development and cancer. Nat. Rev. Cancer. 12, 387-400 (2012).

46. Kalluri, R. \& Weinberg, R. A. The basics of epithelial-mesenchymal transition. J. Clin. Invest. 119, 1420-1428 (2009).

47. Shibue, T. \& Weinberg, R. A. Integrin betal-focal adhesion kinase signaling directs the proliferation of metastatic cancer cells disseminated in the lungs. Proc. Natl Acad. Sci. USA 106, 10290-10295 (2009).

48. Feng, M. et al. Therapeutically targeting glypican-3 via a conformation-specific single-domain antibody in hepatocellular carcinoma. Proc. Natl Acad. Sci. USA 110, E1083-E1091 (2013).

49. $\mathrm{Xu}, \mathrm{M}$. Z. et al. AXL receptor kinase is a mediator of YAP-dependent oncogenic functions in hepatocellular carcinoma. Oncogene 30, 1229-1240 (2011).

50. Hui, K. M. Human hepatocellular carcinoma: expression profiles-based molecular interpretations and clinical applications. Cancer Lett. 286, 96-102 (2009).

51. Choi, H. Y. et al. APP interacts with LRP4 and agrin to coordinate the development of the neuromuscular junction in mice. Elife 2, e00220 (2013).
52. Chen, L. et al. Recoding RNA editing of AZIN1 predisposes to hepatocellular carcinoma. Nat. Med. 19, 209-216 (2013).

53. Neuhuber, B. \& Daniels, M. P. Targeting of recombinant agrin to axonal growth cones. Mol. Cell. Neurosci. 24, 1180-1196 (2003).

54. Swa, H. L., Blackstock, W. P., Lim, L. H. \& Gunaratne, J. Quantitative proteomics profiling of murine mammary gland cells unravels impact of annexin-1 on DNA damage response, cell adhesion, and migration. Mol. Cell. Proteomics 11, 381-393 (2012).

55. Chakraborty, S., ValiyaVeettil, M., Sadagopan, S., Paudel, N. \& Chandran, B. c-Cbl-mediated selective virus-receptor translocations into lipid rafts regulate productive Kaposi's sarcoma-associated herpesvirus infection in endothelial cells. J. Virol. 85, 12410-12430 (2011).

\section{Acknowledgements}

This study was funded in part from Early Career Research grant, Institute of Molecular and Cell Biology (IMCB), Singapore to S.C., and Agency for Science, Technology and Research (A*STAR) funds to W.H. We sincerely thank Dr Matthew P. Daniels, National Institutes of Health, Bethesda, MD, USA for providing Agrin-GFP constructs. We thank Dr Qi Zeng, IMCB, Singapore for sharing additional patient samples and reading the manuscript. We acknowledge the assistance of Mr. Sean Nair, a summer intern in the project. We also thank the Advanced Molecular Pathology Lab at IMCB, Singapore for assistance in immunohistochemical staining.

\section{Author contributions}

S.C. and W.H. conceived and designed the project; S.C., H.L.F.S. and J.G. performed quantitative proteomics analysis with inputs from J.G.; M.L., S.C. and S.C.A. performed in vivo experiments with inputs from W.H. and V.T.; S.C. along with J.C., X.Z., Y.S.O. and L.S.L. performed other experiments; K.M.H. provided HCC patient tissues and plasma samples; and S.C. analysed data and wrote the paper with inputs from W.H.

\section{Additional information}

Supplementary Information accompanies this paper at http://www.nature.com/ naturecommunications

Competing financial interests: S.C. and W.H. have filed a Singapore patent no.10201402829Q for the use of Agrin as a diagnostic marker and therapeutic target for HCC. The remaining authors declare no competing financial interests.

Reprints and permission information is available online at http://npg.nature.com/ reprintsandpermissions/

How to cite this article: Chakraborty, S. et al. An oncogenic role of Agrin in regulating focal adhesion integrity in hepatocellular carcinoma. Nat. Commun. 6:6184 doi: $10.1038 /$ ncomms7184 (2015)

(c) (i) This work is licensed under a Creative Commons Attribution 4.0 cc) International License. The images or other third party material in this article are included in the article's Creative Commons license, unless indicated otherwise in the credit line; if the material is not included under the Creative Commons license, users will need to obtain permission from the license holder to reproduce the material. To view a copy of this license, visit http://creativecommons.org/licenses/by/4.0/ 